ARTICLE

\title{
Raptor determines $\beta$-cell identity and plasticity independent of hyperglycemia in mice
}

\author{
Qinglei Yin ${ }^{1,4}$, Qicheng Ni (10 ${ }^{1,4}$, Yichen Wang ${ }^{1,4}$, Hongli Zhang ${ }^{2}$, Wenyi Li ${ }^{1}$, Aifang Nie ${ }^{1}$, Shu Wang ${ }^{1}$, Yanyun Gu1 , \\ Qidi Wang ${ }^{1,3,5} \otimes$ \& Guang Ning ${ }^{1,5} \bowtie$
}

Compromised $\beta$-cell identity is emerging as an important contributor to $\beta$-cell failure in diabetes; however, the precise mechanism independent of hyperglycemia is under investigation. We have previously reported that $\mathrm{mTORC1/Raptor} \mathrm{regulates} \mathrm{functional} \mathrm{maturation} \mathrm{in}$ $\beta$-cells. In the present study, we find that diabetic $\beta$-cell specific Raptor-deficient mice ( $\beta$ RapKOGFP) show reduced $\beta$-cell mass, loss of $\beta$-cell identity and acquisition of $\alpha$-cell features; which are not reversible upon glucose normalization. Deletion of Raptor directly impairs $\beta$-cell identity, mitochondrial metabolic coupling and protein synthetic activity, leading to $\beta$-cell failure. Moreover, loss of Raptor activates $\alpha$-cell transcription factor MafB (via modulating $\mathrm{C} / \mathrm{EBP} \beta$ isoform ratio) and several $\alpha$-cell enriched genes i.e. Etv1 and Tspan12, thus initiates $\beta$ - to $\alpha$-cell reprograming. The present findings highlight mTORC1 as a metabolic rheostat for stabilizing $\beta$-cell identity and repressing $\alpha$-cell program at normoglycemic level, which might present therapeutic opportunities for treatment of diabetes.

\footnotetext{
${ }^{1}$ Shanghai National Clinical Research Center for Endocrine and Metabolic Diseases, Key Laboratory for Endocrine and Metabolic Diseases of the National Health Commission of the PR China, Shanghai Institute of Endocrine and Metabolic Diseases, Ruijin Hospital, Shanghai Jiao Tong University School of Medicine, 200025 Shanghai, China. ${ }^{2}$ Department of Endocrinology, Seventh People's Hospital of Shanghai University of Traditional Chinese Medicine, 200137 Shanghai, China. ${ }^{3}$ Sino-French Research Center for Life Sciences and Genomics, Ruijin Hospital Affiliated to Shanghai Jiao Tong University School of Medicine, 200025 Shanghai, China. ${ }^{4}$ These authors contributed equally: Qinglei Yin, Qicheng Ni, Yichen Wang. ${ }^{5}$ These authors jointly supervised this work: Qidi Wang, Guang Ning. ${ }^{凶}$ email: wqd11094@rjh.com.cn; guangning@medmail.com.cn
} 
central feature of type 2 diabetes (T2D) is progressive loss of functional $\beta$-cell mass, which ultimately results in $\beta$-cell failure and insulin insufficiency to meet metabolic demands ${ }^{1}$. The development of $\beta$-cell failure can be formulated into three stages: (1) healthy state, (2) impaired coupling of cellular metabolism with insulin secretion, and finally (3) loss of features as a mature, hormone-laden cell (dedifferentiation) with conversion to other endocrine cell types ${ }^{2}$. It was proposed that loss of $\beta$-cell identity might be one of the mechanisms of loss of functional $\beta$-cell mass in diabetes ${ }^{3,4}$. Deletion of several important transcription factors, i.e. Foxo1 (ref. ${ }^{5}$ ), $P d x 1$ (ref. $^{6}$ ), $N k x 6.1$ (ref. ${ }^{7}$ ), MafA ${ }^{8}, N k x 2.2$ (ref. ${ }^{9}$ ), Pax6 (ref. ${ }^{10}$ ) perturbs $\beta$-cell identity by silencing $\beta$-cell functional genes and induction of genes characteristic of other islet cell types. It has been suggested that metabolic inflexibility is a key step of $\beta$-cell dedifferentiation and $\beta$-cell failure 2,11 . Interestingly, $\beta$-cell dedifferentiation and reprogramming appeared to be reversible upon normalization of glucose levels ${ }^{12,13}$. Recently, we have reported that $\beta$-cells are dedifferentiated in T2D patients with adequate glucose control and non-diabetic chronic pancreatitis, suggesting dedifferentiation can be a cause of $\beta$-cell failure, not merely as a consequence of hyperglycemia $^{14}$. It still remains unclear whether certain signal pathway controls compromised $\beta$-cell identity, independent of hyperglycemia.

mTOR is an evolutionarily conserved, nutrient-sensing serine-threonine protein kinase, functioning in the form of at least two large protein complexes, mTOR complex 1 (mTORC1) and mTOR complex 2 (mTORC2) ${ }^{15,16}$. mTORC1 consists of RAPTOR (regulatory associated protein of mTOR), mLST8, PRAS40, DEPTOR, and mTOR, which is sensitive to Rapamycin 17,18 . Recent studies have shown that mTORC1 activity was upregulated in islets from $\mathrm{db} / \mathrm{db}$ mice and human of $\mathrm{T} 2 \mathrm{D}$, indicating its critical role in adaptation and decompensation during diabetes progression 19,20 . The extensive research revealed that physiological mTORC1 activation is essential for $\beta$-cell development, growth, function, and survival ${ }^{21,22}$, whereas its sustained over-activation might lead to $\beta$-cell failure ${ }^{23,24}$. Recently, we have reported that $\beta$-cell specific Raptor-deficient mice disrupts postnatal $\beta$-cell growth and functional maturation, at least partly due to changes in DNA methylation pattern ${ }^{25}$. Later with the same knockout mice, Blandino-Rosano et al. ${ }^{26}$ found S6K signaling had more impact on $\beta$-cell size and apoptosis, while $4 \mathrm{E}-\mathrm{BP} 2 / \mathrm{eIF} 4 \mathrm{E}$ activity was more involved in $\beta$-cell proliferation. However, the above observations were all collected in diabetic conditions, which made it obscure to which extent hyperglycemia per se underlies or exacerbates alterations in $\beta$-cell phenotype in mTORC1-deficient mice. Interestingly, in diabetic Raptor-KO islet, we observed decreased expression of $\beta$-cell identity genes and upregulation of progenitor markers. This work builds on our previous study, but takes it further by dissecting high glucose from gene deletion to explore the exact role of mTORC1 on $\beta$-cell identity and plasticity.

In the present study, we use 4 -week-old $\beta$-cell lineage tracing Raptor-KO mice, keep their blood glucose at normal range for 4 weeks by implanting insulin pellets immediately after the onset of diabetes and then trace the fates of $\beta$-cells. Our results demonstrate that mTORC1 directly regulates adult $\beta$-cell identity, mitochondrial metabolic coupling, and protein synthetic activity to maintain mature $\beta$-cell differentiated phenotype. Moreover, Raptor is required for $\beta$-cell to suppress $\alpha$-cell enriched genes, including a-cell transcription factor $M a f B$, and a group of $\alpha$-cell enriched genes, i.e. Etv1, Tspan 12 and thus prevent $\beta$ - to $\alpha$-cell reprograming at normal glucose range. Our data highlight mTORC1 signaling as an underlying mechanism implicated in promoting the terminal differentiation of $\beta$-cells and repressing $\beta$-cell default.

\section{Results}

Increased $\boldsymbol{\alpha} / \boldsymbol{\beta}$-cell ratio in $\boldsymbol{\beta}$ RapKO ${ }^{\mathrm{GFP}}$ mice. Recently, we have reported that Raptor regulates functional maturation in murine $\beta$ cells ${ }^{25}$. The heatmap showed that loss of Raptor reduced the expressions of genes critical to $\beta$-cell $(P d x 1, N k x 6.1$, NeuroD1, Foxo1, Isl1), whereas the expressions of $\alpha$-cell key factors (Irx2, Arx, MafB, Pou6f2, Fev, Kcnj3, and $S v 2 b$ ) and progenitor markers (Neurog3, Gata6, Hnf4a, Notch1, and Hes1) (Fig. 1a) were strongly upregulated in mutant islets. These data suggest that Raptor-deficient immature $\beta$-cells fail to maintain $\beta$-cell identity and adopt progenitor-like features with the tendency to convert to $\alpha$-cells.

To elucidate the role of mTORC1 in maintaining $\beta$-cell identity, we deleted Raptor which is an essential and specific component of mTORC1 in $\beta$-cells and traced their fates using a Rosa26 ${ }^{G F P}$ lineage labeling. This was achieved by generating RIPCre; Raptor ${ }^{-/}{ }^{-}$Rosa26GFP $\left(\beta \mathrm{RapKO}^{\mathrm{GFP}}\right.$ ) mice and their control littermates RIP-Cre; Raptor ${ }^{+/+}$; Rosa26 GFP (WT) (Supplementary Fig. 1a). GFP expression was exclusively detected in the insulinproducing cells in the pancreas of RIPCre-Rosa26 ${ }^{G F P}$ mice (Supplementary Fig. 1b) and $\mathrm{GFP}^{+}$cells can be obtained by fluorescence-activated cell sorting (FACS) (Supplementary Fig. 1c). The Raptor mRNA level was almost undetectable in $\beta$ cells but was abundantly expressed in other tissues such as heart, kidney, muscle, liver, and hypothalamus (Supplementary Fig. 1d). The islets isolated from $\beta$ RapKOGFP mice showed reduced expression of RAPTOR and de-phosphorylation of mTORC1 targets PS6 (Ser240/244) and 4E-BP1 (shift from the highly phosphorylated $\gamma$-band to the non-phosphorylated $\alpha$-band and an intermediate $\beta$-band) (Supplementary Fig. 1e). Moreover, loss of mTORC1 activity (PS6 Ser240/244) could only be detected in insulin-positive $\left(\mathrm{Ins}^{+}\right)$cells of dispersed mutant islets (Supplementary Fig. 1f).

$\beta$ RapKOGFP mice started to display elevated random and $6 \mathrm{~h}$ fasting blood glucose levels at the age of 4 weeks (Supplementary Fig. $2 \mathrm{a}, \mathrm{b}$ ), and they developed overt diabetes at the age of 8 weeks when challenged with intraperitoneal glucose injection (Supplementary Fig. 2c). The diabetic phenotype was in line with our previous observations on $\beta$ RapKO mice ${ }^{25}$. We found approximately $70 \%$ reduction in $6 \mathrm{~h}$ fasting plasma insulin levels (Supplementary Fig. 2d), but not in $6 \mathrm{~h}$ fasting glucagon concentrations (Supplementary Fig. 2e) in 8-week-old $\beta$ Rap$\mathrm{KO}^{\mathrm{GFP}}$ mice. Accordingly, the Ins ${ }^{+}$cells per islet (Fig. 1b) and $\beta$ cell mass (Supplementary Fig. 2f) were significantly reduced in $\beta$ RapKOGFP mice. Importantly, we detected that $\mathrm{Gcg}^{+}$cells per islet were significantly increased $(13.98 \pm 0.61$ vs $11.43 \pm 0.37$ in WT, $p<0.05$, Fig. 1c) in $\beta$ RapKO ${ }^{\text {GFP }}$ islets. Although the $\alpha$-cell mass only tended to increase (Supplementary Fig. $2 \mathrm{~g}$ ), the $\alpha$-cell mass $/(\alpha+\beta)$-cell mass was doubled in $\beta$ RapKO GFP mice (Fig. 1d). Interestingly, the increased $\alpha$-cells were not derived from the proliferation of themselves, as quantitative analysis revealed that the percentage of $\mathrm{Ki}^{+} 7^{+} / \mathrm{Gcg}^{+}$cells remained unaltered in mutant islets (Fig. 1e). At 12 weeks of age, further decreases in $\mathrm{Ins}^{+}$cells $(43.23 \pm 3.36$ vs $57.01 \pm 3.48$ in WT, $p<0.05)$ and increases in $\mathrm{Gcg}^{+}$cells $(22.04 \pm 1.72$ vs $13.35 \pm 0.69$ in WT, $p<0.05)$ were detected in $\beta$ RapKOGFP islets (Supplementary Fig. $2 \mathrm{~h}$ ).

We then tested whether there existed $\beta$ to $\alpha$ shift upon loss of Raptor. We performed co-immunostaining for GFP, insulin, and glucagon on pancreatic sections from 8-week-old WT and $\beta$ RapKOGFP mice. We found significantly increased number of Raptor-deficient GFP cells coexpressing glucagon in mutant islets $(6.65 \% \pm 0.27$ vs $0.10 \% \pm 0.008$ in WT, $p<0.001)$ (Fig. 1f, g). However, we did not find evidence of $\beta$-cell reprogramming to $\delta$ or PP cells: neither somatostatin nor pancreatic polypeptide was co-localized with GFP/Ins ${ }^{+}$in $\beta$ RapKO ${ }^{\mathrm{GFP}}$ islet (Supplementary Fig. $2 \mathrm{i}, \mathrm{j}$ ). These results suggest that Raptor-deficient $\beta$-cells lose their identities and adopt $\alpha$-cell fates. 
a

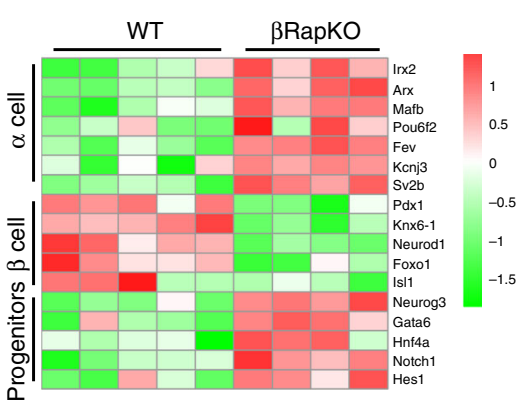

b

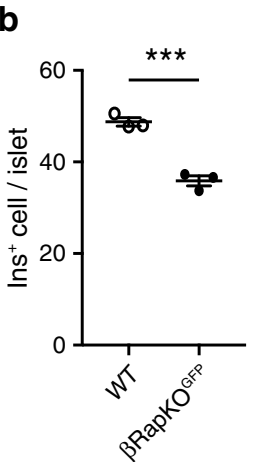

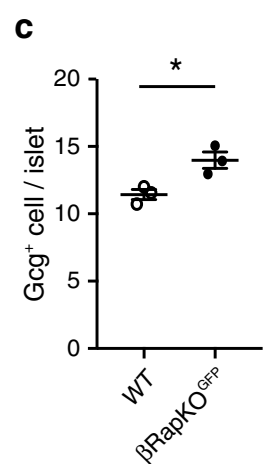

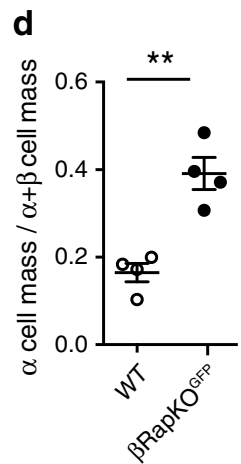

e

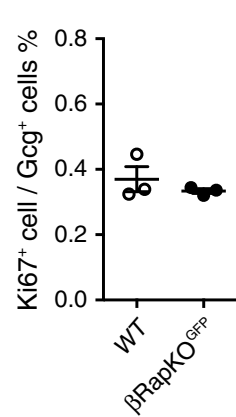

f
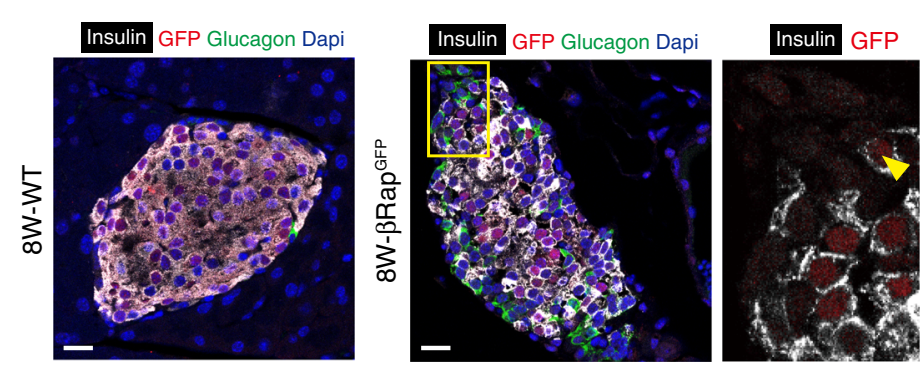

GFP Glucagon
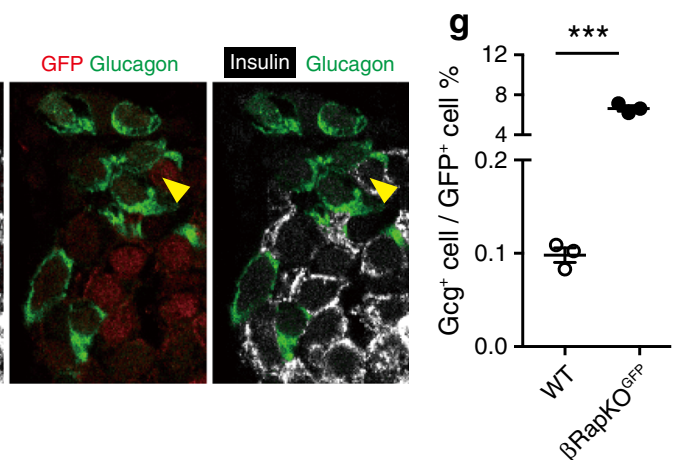

h
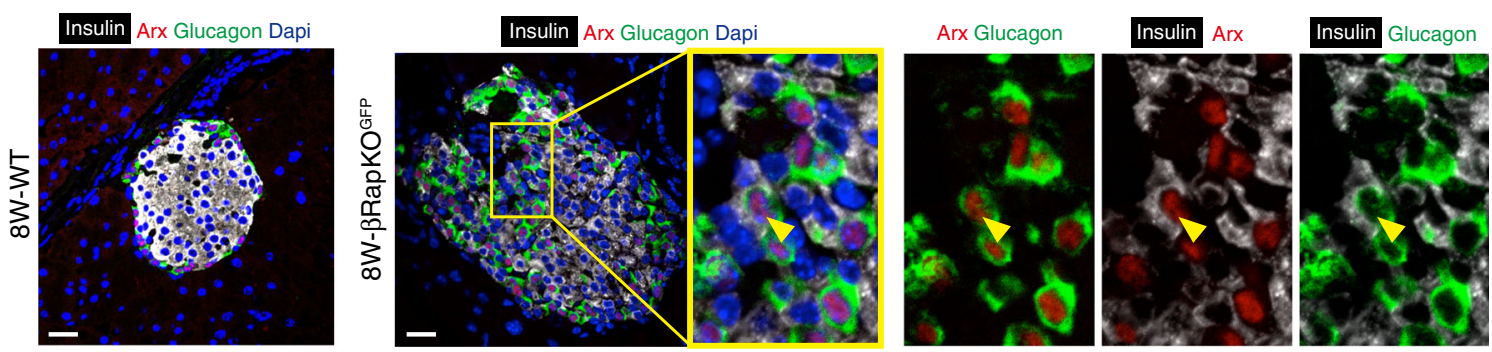

i

Insulin MafB Glucagon Dapi
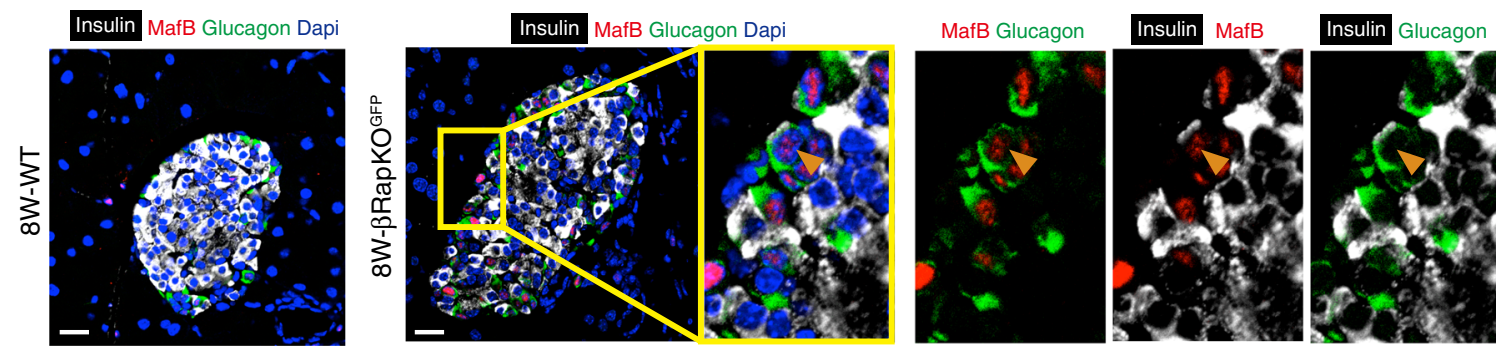

j

$\alpha$ cell

$\beta$ cell
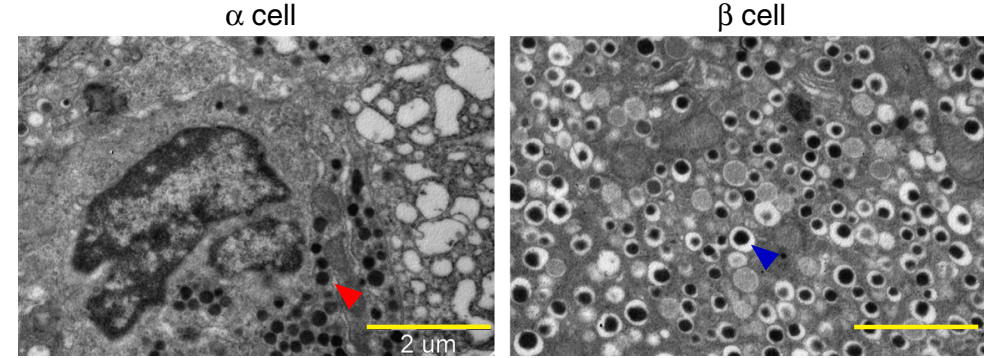

8W- $\beta$ RapKO GFP $\beta$ cell

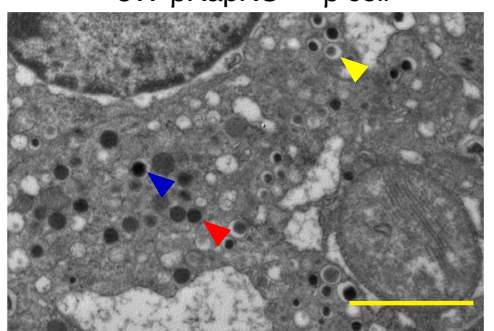

If $\beta$-cells were the source of newly formed $\alpha$-cells, we hypothesized that, during the transition phase, the transcription factors crucial for $a$-cell might be expressed in these multihormonal GFP cells. Indeed, we detected the expression of $\alpha$-cell specific transcription factors Arx (Fig. 1h, Supplementary Fig. 3a) and/or MafB (Fig. 1i, Supplementary Fig. 3b) in Ins ${ }^{+} \mathrm{Gcg}^{+}$cells in 8-week-old $\beta$ RapKOGFP islets. Moreover, the multi-hormonal cells included both $\mathrm{Arx}^{+}$and $\mathrm{Arx}^{-}$subpopulations (Supplementary Fig. 3c), representing different converted stages during transition. Interestingly, we also identified expressions of Glut2 (Supplementary Fig. 3d) and/or Pdx1 (Supplementary Fig. 3e) in some $\mathrm{Ins}^{+} \mathrm{Gcg}^{+}$cells, indicating some multi-hormonal cells 


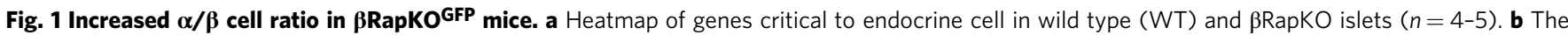
number of $\mathrm{Ins}^{+}$cells per islet $(n=3, p=0.0008)$ and $\mathbf{c}$ the number of $\mathrm{Gcg}^{+}$cells per islet were calculated $(n=3, p=0.023)$. At least, 46 islets were used for quantifications. d The proportion of pancreatic $\alpha$-cell mass among pancreatic $\alpha$ - and $\beta$-cell mass was shown $(n=4, p=0.0017)$. e The proliferation of $\mathrm{Gcg}^{+}$cell was determined by quantification of the percentage of $\mathrm{Ki}^{+}{ }^{+}$cells in $\mathrm{Gcg}^{+}$cells $(n=3)$. At least, 50 islets were used for quantifications. $\mathbf{f}$ Pancreatic sections from 8-week-old WT, $\beta$ RapKO GFP mice were immunostained for insulin (white), glucagon (green), and GFP (red). Nuclei were stained with DAPI (blue) $(n=3)$. The yellow arrows indicated insulin, glucagon, and GFP co-stained cells. Scale bars, $20 \mu \mathrm{m}$. $\mathbf{g}$ The percentage of GFP+Gcg ${ }^{+}$cells among GFP+ cells in 8-week-old WT and $\beta$ RapKOGFP mice $(n=3, p=1.83046 \mathrm{E}-05)$. At least, $1837 \mathrm{GFP}+$ cells over three mice each group were used for quantifications. $\mathbf{h}$ Representative pancreatic sections showed expression of $\alpha$-cells marker-Arx (red), insulin (white), and glucagon (green) in WT and $\beta$ RapKOGFP mice at 8 weeks of age $(n=3)$. Scale bars, $20 \mu \mathrm{m}$. i Immunostaining showed expression of $\alpha$-cells marker-MafB (red), insulin (white), and glucagon (green) in control and $\beta$ RapKOGFP mice at 8 weeks of age $(n=3)$. Yellow boxes showed the specific areas of the islet, which were enlarged and represented by arrows on the right to demonstrate protein expression within specific cells. Scale bars, $20 \mu \mathrm{m}$. $\mathbf{j}$ Transmission electron microscopy (TEM) of pancreatic islets reveals a combination of mature insulin, immature insulin, and glucagon-like granules in the same cell in $\beta$ RapKOGFP mice $(n=3$ independent samples). Granule identity is indicated by colored arrows: glucagon (red), mature insulin (blue), and immature insulin (yellow). Data represent means \pm SEM. ${ }^{\star} p<0.05,{ }^{\star \star} p<0.01,{ }^{\star \star \star} p<0.001$ by two-sided Student's $t$-test.

retained expression of $\beta$-cell crucial genes. These "mixed phenotype" cells provide evidence that Raptor knockout $\beta$-cells achieve a-like features.

Electron microscopy was also performed on 8-week-old WT and $\beta$ RapKO GFP islets. The light microscopy showed that intact WT mature $\beta$-cells display typical insulin granules with characteristic electron-dense insulin crystal cores surrounded by a clear "halo" (Fig. 1j, middle panel, blue arrow), whereas glucagon-containing granules in a-cells lack any such "halo" (Fig. 1j, left panel, red arrow). In contrast, we observed a few Raptor-deficient $\beta$-cells had mixed features of $\alpha$ - and $\beta$-granules, some with dark (mature insulin granule, Fig. $1 j$, right panel, blue arrow) or light electron-dense crystal cores (immature insulin granule, Fig. 1j, right panel, yellow arrow) and others lost their outer "halo" (Fig. 1j, right panel, red arrow). These findings further support that Raptor-deficient $\beta$-cells have morphological features of $\alpha$-like cells.

Impaired proinsulin synthesis and ATP levels for GSIS. The occurrence of hyperglycemia in diabetes can diminish $\beta$-cell function and alter $\beta$-cell identity ${ }^{27}$. To determine the independent impact of Raptor and hyperglycemia on $\beta$-cell identity and function, we implanted slow-release insulin pellet on 4-week-old $\beta$ RapKOGFP mice (the age when fasting blood glucose levels started to rise) for 4 weeks and kept the serum blood glucose at normal levels in mutant rodents (Fig. 2a). As expected, implantation of insulin pellet (releasing $0.2-0.3 \mathrm{U}$ per day) caused a rapid fall in random blood glucose from $12.86 \pm 0.37$ to $5.43 \pm$ $0.96 \mathrm{mM}$ on the day of implantation, 2 days later to $8.92 \pm 0.80$ $\mathrm{mM}$ (Fig. 2b). Afterwards, insulin-treated $\beta$ RapKO ${ }^{\mathrm{GFP}}$ mice (euglycemic $\beta$ RapKO ${ }^{\mathrm{GFP}}$ ) maintained normoglycemia for 4 weeks, with comparable blood glucose levels as that of WT mice, whereas untreated mutant mice (diabetic $\beta$ RapKOGFP) exhibited severe hyperglycemia (Fig. 2b). Insulin treatment for 4 weeks partially prevented the change in islet morphology (Fig. 2c) and restored MafA expression in mutant mice (Supplementary Fig. 4a). On the contrary, the severely reduced expression levels of maturity marker UCN3 observed in diabetic $\beta$ RapKOGFP islet $^{25}$ (Fig. 2d) were not improved in euglycemic mutants (Fig. 2d). It is known that Ucn3 is exclusively expressed in mature $\beta$-cells and is required for full glucose-stimulated insulin secretion in mouse islet ${ }^{28,29}$. We then performed GSIS in isolated islets from the three groups and measured C-peptide levels to obviate assessment of exogenous insulin. Compared to WT, both diabetic and euglycemic $\beta$ RapKOGFP islets exhibited increased glucose threshold for C-peptide secretion: i.e. elevated basal C-peptide secretion, but significantly diminished glucosestimulated C-peptide secretion at $16.7 \mathrm{mM}$ glucose (Fig. 2e). The diminished stimulation index (fold change in GSIS) in euglycemic mutant islet unveiled that Raptor itself was the cause of impaired C-peptide secretory response to glucose (Fig. 2e). In parallel, the islet ATP content was comparable at basal glucose concentrations among the three groups, whereas acute glucose-stimulated ATP increase was significantly reduced $(p<0.05)$ in both diabetic and euglycemic $\beta$ RapKO GFP mice (Fig. $2 \mathrm{f}$ ).

Intriguingly, though pancreatic C-peptide content in euglycemic $\beta$ RapKOGFP was completely restored (Fig. $2 \mathrm{~g}$ ), pancreatic proinsulin content was still markedly reduced compared to WT (Fig. 2h), indicating impaired proinsulin biosynthetic activity in $\beta$-cell is the main defect due to loss of Raptor. It has previously been suggested that Raptor deficiency impairs proinsulin processing by impairing the synthesis of Carboxypeptidase $\mathrm{E}$ $(\mathrm{CPE})^{26}$. We found the proinsulin/C-peptide ratio of both pancreatic tissue (Fig. 2i) and cultured islets (at 2.8 and 16.7 $\mathrm{mM}$ glucose) (Fig. 2j) were significantly increased in diabetic $\beta$ RapKOGFP mice, but were completely reversed in euglycemic $\beta$ RapKOGFP mice. We further performed double staining of insulin and $\mathrm{CPE}$ on 8 - and 2-week-old mutant pancreas. Interestingly, we found that the expression of CPE was decreased in diabetic $\beta$ RapKO ${ }^{\mathrm{GFP}}$ mice, while in euglycemic $\beta$ RapKO ${ }^{\mathrm{GFP}}$ mice the expression of CPE was reversed (Supplementary Fig. 4b). Moreover, in 2-week-old euglycemic $\beta$ RapKOGFP mice, CPE expression was comparable to that of WT controls (Supplementary Fig. 4c). To further test the specific effect of mTORC1 on CPE expression and proinsulin/insulin ratio, we silenced Raptor in INS-1 cells. Knockdown of Raptor dramatically reduced both proinsulin and insulin abundance shown in immunoblot, but presented a comparable proinsulin/insulin ratio in these two groups (Fig. 2k). Moreover, we did not detect difference in mRNA expression of processing genes (Pcsk1, Pcsk2, and CPE) (Supplementary Fig. 4d) and protein abundance of CPE (Supplementary Fig. 4e) between SiNC and SiRaptor conditions. Taken together, our data demonstrate that loss of Raptor had detrimental impacts on proinsulin production, metabolic coupling of insulin secretion in $\beta$-cells, but the abnormal increase in proinsulin/insulin ratio and defects in CPE expression were the consequences of hyperglycemia.

Impaired metabolic coupling of insulin secretion. To identify Raptor-regulated pathways in $\beta$-cells, we conducted RNAsequencing analysis on FACS-sorted $\mathrm{GFP}^{+}$cells obtained from 8 -week-old WT, diabetic, and euglycemic $\beta$ RapKO ${ }^{\mathrm{GFP}}$ mice. After filtering out the repeated genes, we obtained a list of 437 differentially expressed genes $\geq 1.5$-fold change and with $p<0.05$ for at least one of the possible comparisons between two groups. Within these 437 genes, the number of differentially expressed genes for WT vs diabetic $\beta$ RapKO GFP was 334 , WT vs euglycemic $\beta$ RapKO ${ }^{\mathrm{GFP}}$ was 113 , and diabetic $\beta$ RapKO ${ }^{\mathrm{GFP}}$ vs euglycemic 
a

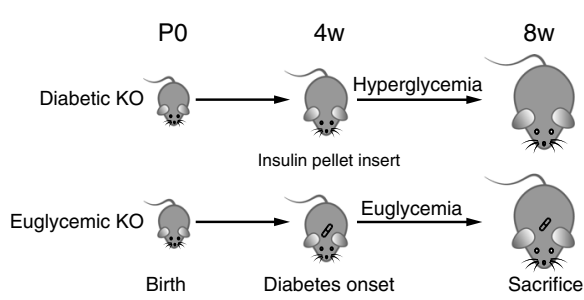

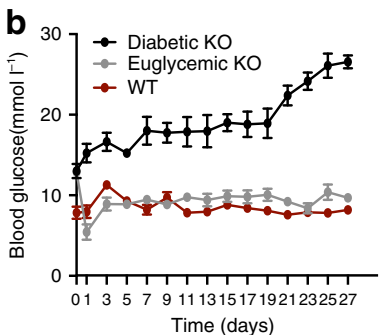

C

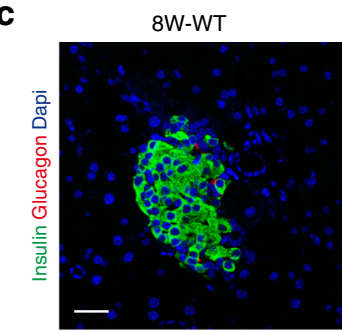

8W-diabetic KO

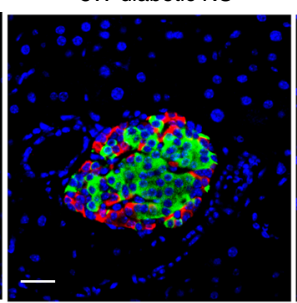

d
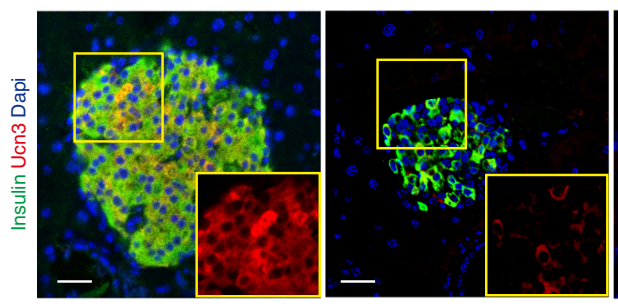

e
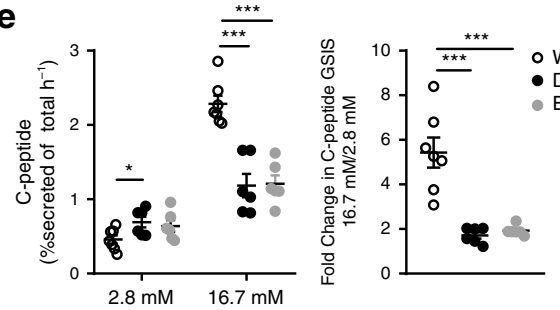

g

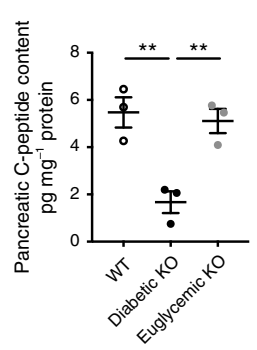

h

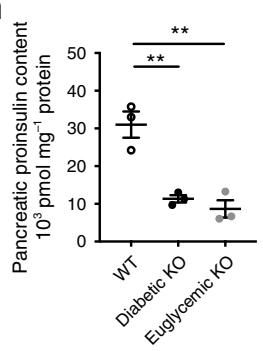

8W-euglycemic KO
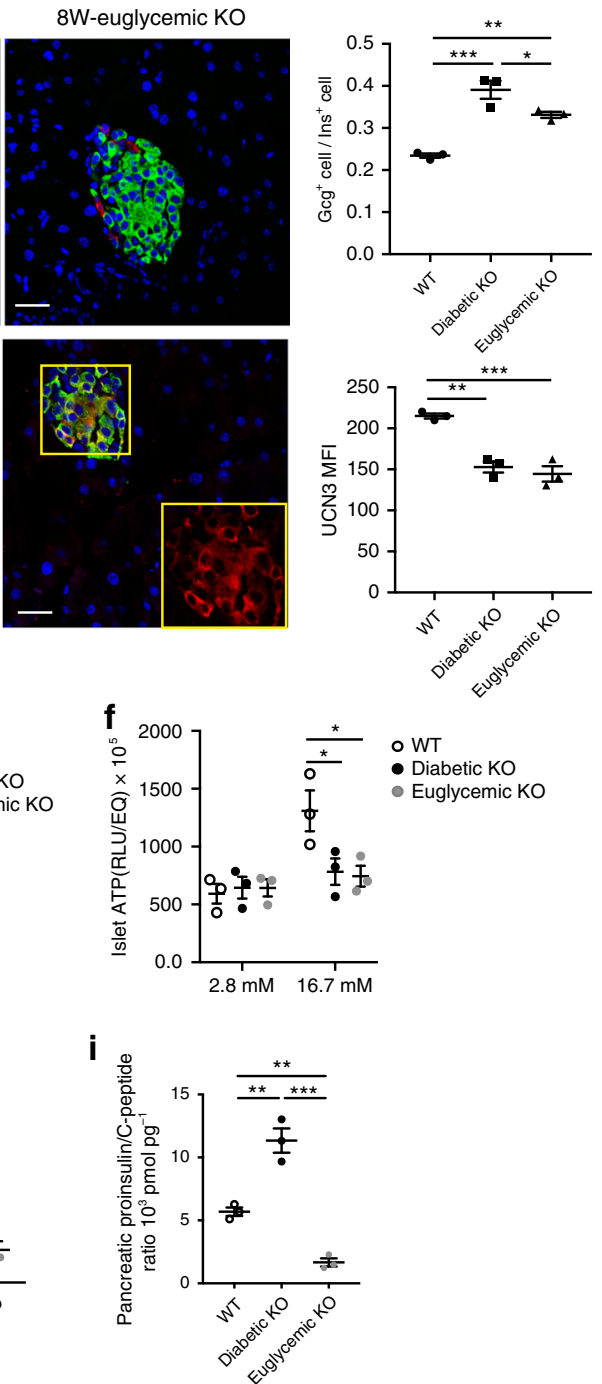

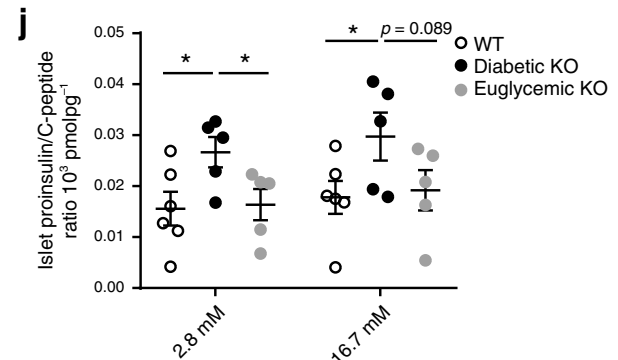

$\beta$ RapKOGFP was 61 (Fig. 3a). We then subjected the differentially expressed genes regulated by Raptor to hierarchical clustering and found that diabetic and euglycemic $\beta$ RapKO ${ }^{\mathrm{GFP}}$ groups were most closely clustered, whereas diabetic/euglycemic $\beta$ RapKO GFP and WT were distantly clustered (Fig. 3b). The limited number of changed genes and closely clustering between diabetic and $\mathbf{k}$

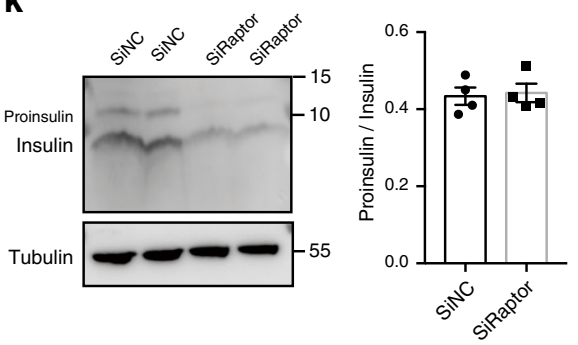


Fig. 2 Impaired proinsulin synthesis and ATP levels for GSIS. a Schematic of insulin pump implantation. b Random blood glucose levels were monitored every other day in WT, diabetic $\beta$ RapKOGFP, and euglycemic $\beta$ RapKOGFP mice from 4 weeks to 8 weeks old $(n=6$ for WT, $n=7$ for diabetic KO, $n=13$ for euglycemic KO). c Representative pancreatic sections immunostained for insulin (green) and glucagon (red). The ratio of $\mathrm{Gcg}^{+}$cells to Ins ${ }^{+}$cells was calculated ( $n=3$ ). At least 50 islets or 2000 insulin-positive cells were used for quantifications. Scale bars, $20 \mu \mathrm{m}$. $\mathbf{d}$ Images of islets labeled with insulin (green) and UCN3 (red). MFI of UCN3 in these three groups $(n=3)$. At least 10 islets from three sections were used for MFI. Yellow boxes showed the expression of UCN3. Scale bars, $20 \mu \mathrm{m}$. e In vitro glucose-stimulated C-peptide secretion in islets at 2.8 and $16.7 \mathrm{mM}$ glucose levels for $1 \mathrm{~h}$ (reported as percent of $C$-peptide content) ( $n=7$ for WT, $n=6$ for diabetic KO, $n=6$ for euglycemic KO). White, black, and gray circles represented the WT, diabetic $\beta$ RapKOGFP, and euglycemic $\beta$ RapKOGFP groups, respectively. Stimulation index of C-peptide secretion in diabetic $\beta$ RapKO GFP and euglycemic $\beta$ RapKO GFP mice as compared with controls ( $n=7$ for WT, $n=6$ for diabetic KO, $n=6$ for euglycemic KO). f ATP content at $2.8 \mathrm{mM}$ glucose and $16.7 \mathrm{mM}$ glucose ( $n$ 3). $\mathbf{g}$ Pancreatic C-peptide content ( $n=3$ independent samples) and $\mathbf{h}$ pancreatic proinsulin content normalized by protein concentration were shown ( $n$ $=3$ independent samples). $\mathbf{i}$ The ratio of pancreatic proinsulin to C-peptide content was determined ( $n=3$ independent samples). $\mathbf{j}$ The ratio of islet proinsulin to C-peptide content per 10 size-matched islets at $2.8 \mathrm{mM}$ and high $16.7 \mathrm{mM}$ glucose levels was determined ( $n=6$ for WT, $n=5$ for diabetic $\mathrm{KO}, n=5$ for euglycemic KO). k INS-1 cells were transfected with SiRaptor or SiNC for $72 \mathrm{~h}$. Immunoblotting and quantification of insulin and proinsulin in INS-1 cells transfected with SiNC or SiRaptor ( $n=4$ independent cell experiments). Data represent means \pm SEM. ${ }^{\star} p<0.05,{ }^{\star \star} p<0.01,{ }^{\star \star \star} p<0.001$ by twosided Student's t-test and one-way ANOVA adjusted by LSD multiple comparison, $p$ values included in source data.

critical for $\beta$-cell function, i.e. response to glucose, insulin secretion, glucose metabolic process, secretory granule, vesicle/ion transport, and pancreas development (Fig. 3c).

The transcriptome changes from the RNA-seq encompassed virtually key pathways required for physiologic insulin secretion, which allowed us to formulate a hypothesis on the physiologic role of Raptor in $\beta$-cells (Fig. 3d). The genes required for fuel metabolism (Glut2, Idh2, Atp4a, Cox6a2, mt-Co3, mt-Co1, mt$N d 2$, Nqo1, Ffar1) were all significantly downregulated in both diabetic and euglycemic Raptor-deficient $\beta$-cells (Fig. 3d). In addition to mitochondrial dysfunction, loss of Raptor as previously reported ${ }^{25}$ caused upregulation of disallowed genes Ldha, $H k 1$, and $A l d o B$, which could not be rescued after glucose normalization (Fig. 3d). Moreover, secretory pathway (Gipr, Trpm5, Btg2, MaoB, Fkbp1b) and granule trafficking (Slc30a8, Sytl4, Rab37) were obviously decreased in both diabetic and euglycemic Raptor-deficient $\beta$-cells (Fig. 3d). On the contrary, some genes involved in anti-inflammatory (Dusp1, Hcar2), eNOS, and ROS (Pecam1, Arg1, CEPBS, Eng, and Aqp11) process were completely reversed in euglycemic $\beta$ RapKO ${ }^{\mathrm{GFP}} \beta$-cells, indicating that inflammatory and ROS pathway were induced by glucotoxicity rather than loss of Raptor.

We confirmed dramatic decreases in glucose transporter Glut2, subunit of the cytochrome $c$ oxidase complex Cox6a2, member of $\mathrm{NAD}(\mathrm{P}) \mathrm{H}$ dehydrogenase (quinone) family $\mathrm{Nqo1}$ in $\beta$-cells from both diabetic and euglycemic $\beta$ RapKOGFP mice using qRT-PCR (Fig. 3e-f). Moreover, the expression levels of Slc30a8 (ZnT8) required for insulin crystallization/secretion and Sytl4, Rab37 participating in insulin granule trafficking and docking were all decreased significantly in both diabetic and euglycemic $\beta$ RapKOGFP $\beta$-cells (Fig. 3e). The ectopically upregulation of disallowed genes $H k 1, A l d o B$, and Ldha in euglycemic $\beta$ RapKOGFP $\beta$-cells were reproduced by qRT-PCR (Fig. $3 g$ ). We further compared our RNA-seq data with the disallowed gene list from Pullen et al. ${ }^{30}$, and found many disallowed genes (i.e. Serpina7, Gm2115, Gucy2c, Aass, Trf, Hmgcs2, Gfra1, Ptprk, Tspan12, and Sh3bgrl2) were upregulated in Raptor-deficient $\beta$ cells (Supplementary Fig. 5). The immunostaining experiment identified that GLUT2 protein expression was significantly decreased in $\beta$ RapKO ${ }^{\mathrm{GFP}}$ islet, and this dramatic change was not reversed in euglycemic mutants (Fig. 3h).

Induction of $\boldsymbol{\alpha}$-cell-enriched genes in euglycemic $\beta$ RapKO ${ }^{\mathrm{GFP}}$. To get a better understanding whether alterations in $\beta$-cell identity and plasticity in Raptor-deficient $\beta$-cells were reversible upon normalization of blood glucose levels, we performed the principal component analysis (PCA) based on two published datasets of signature genes individually enriched in $\beta$ - and $\alpha$ - cells $^{31,32}$. Notably, in the PCA plot, diabetic and euglycemic $\beta$ RapKOGFP groups were most closely clustered, whereas diabetic/euglycemic $\beta$ RapKO ${ }^{\mathrm{GFP}}$ and WT were distantly clustered (Fig. 4a). Based on the reference lists by Qiu et al. ${ }^{31}$ and Cigliola et al. $^{32}$, in purified GFP-sorted $\beta$-cells, Raptor regulated a subset of genes (57 genes) that are highly expressed in $\alpha$-cell lineage, among which $94.7 \%$ were significantly upregulated (Figs. $4 b, c$ ). Importantly, many of these a-cell-enriched genes maintained at high expression levels in euglycemic $\beta$ RapKO GFP $\beta$-cells (Fig. $4 c$ ). By qRT-PCR, we demonstrated that important $\beta$-cell identity genes, $N k x 6.1$ and $P d x 1$ were significantly downregulated, whereas progenitor markers such as Aldh1a3, Ngn3, Sox9 were upregulated in both diabetic and euglycemic $\beta$ RapKO ${ }^{\text {GFP }} \beta$-cells (Fig. 4d, Supplementary Fig. 6a). Moreover, the important $\alpha$-cell gene product glucagon and crucial a-cell transcription factors $M a f B$ and $\operatorname{Ir} x 2$ were significantly upregulated in diabetic $\beta$ RapKOGFP $\beta$-cells and remained at high expression levels in euglycemic $\beta$ RapKO GFP $\beta$-cells (Fig. 4d). Another $\alpha$-cell transcription factor Arx tended to increase in the $\beta$ RapKOGFP group but did not reach statistical significance (Fig. $4 \mathrm{~d}$ ). In addition, several genes important for a-cell, i.e. Pyy, Ppy, Slc38a5, Cadm1, and Ace 2 were all significantly upregulated in purified $\beta$-cells from euglycemic $\beta$ RapKOGFP mice (Fig. 4d, Supplementary Fig. 6a). These results indicate that Raptor determines $\beta$-cell identity and plasticity, independent of hyperglycemia.

We then measured $\mathrm{GFP}^{+}$multi-hormonal cells in the three groups. The percentage of $\mathrm{GFP}^{+} \mathrm{Gcg}^{+}$cells among all $\mathrm{GFP}^{+}$cells in diabetic $\beta$ RapKOGFP were significantly increased $(5.99 \% \pm 0.69$ vs $0.11 \% \pm 0.02$ in WT, $p<0.01)$. The multi-hormonal cells were much less in euglycemic mutants but still remained at a 13 -fold increase compared to WT $(1.32 \% \pm 0.09$ vs $0.11 \% \pm 0.02$ in WT, $p$ $<0.01$ ) (Fig. 4e). We further applied in situ immunostaining against ALDH1A3, a $\beta$-cell dedifferentiation marker, on pancreatic section of the three groups. ALDH1A3 was strongly induced in Ins $^{+}$cells of diabetic $\beta$ RapKO ${ }^{\text {GFP }}$ mice $(37.95 \% \pm 5.06$ vs $0.94 \% \pm 0.06$ in WT, $P<0.05)$ and the high proportion of Aldhla3 ${ }^{+}$Ins $^{+}$cells remained $(35.52 \% \pm 2.88)$ in euglycemic $\beta$ RapKOGFP mice when glycemia has been adequately controlled (Fig. 4f). In addition, we found that some $\beta$-cells from euglycemic $\beta$ RapKO GFP mice still lost $\beta$-cell specific transcriptional factor Nkx6.1 (Supplementary Fig. 6b). Collectively, these results indicate that Raptor itself orchestrates many of transcriptional changes that define fate map for $\beta$-cell before the onset of hyperglycemia, while hyperglycemia accelerates the final decision.

mTORC1 regulates $\alpha$-cell transcriptional factor MafB. We then assessed essential $\alpha$-cell transcriptional factors and glucagon in purified $\beta$-cells from 2 -week-old $\beta$ RapKO ${ }^{\mathrm{GFP}}$ mice and their age- 
a

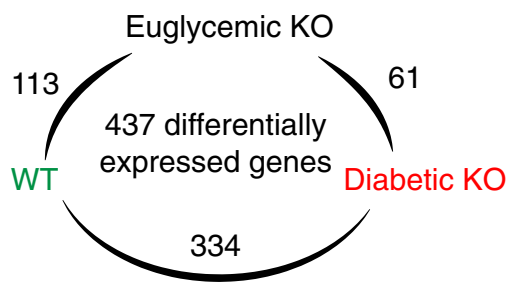

d

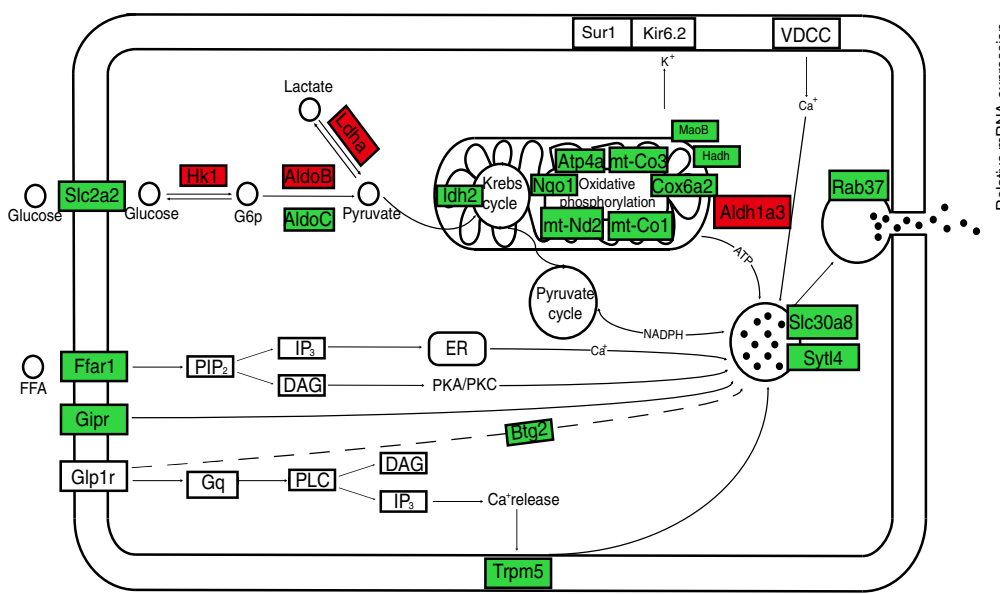

b

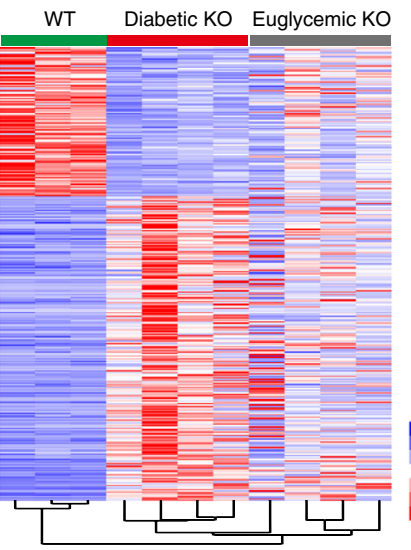

e
C

\begin{tabular}{|lc|}
\hline \multicolumn{2}{|c}{ WT vs euglycemic KO } \\
\hline GO Biological Function & P value \\
\hline response to glucose & $2.36 \mathrm{E}-06$ \\
\hline regulation of insulin secretion & $4.63 \mathrm{E}-06$ \\
\hline response to nutrient levels & $8.46 \mathrm{E}-06$ \\
\hline secretory granule & $7.50 \mathrm{E}-05$ \\
\hline vesicle & $3.32 \mathrm{E}-04$ \\
\hline pancreas development & $3.54 \mathrm{E}-04$ \\
\hline glucose metabolic process & $7.77 \mathrm{E}-04$ \\
\hline regulation of ion transport & $1.53 \mathrm{E}-03$ \\
\hline secretion & $3.11 \mathrm{E}-03$ \\
\hline cell differentiation & 0.12 \\
\hline
\end{tabular}

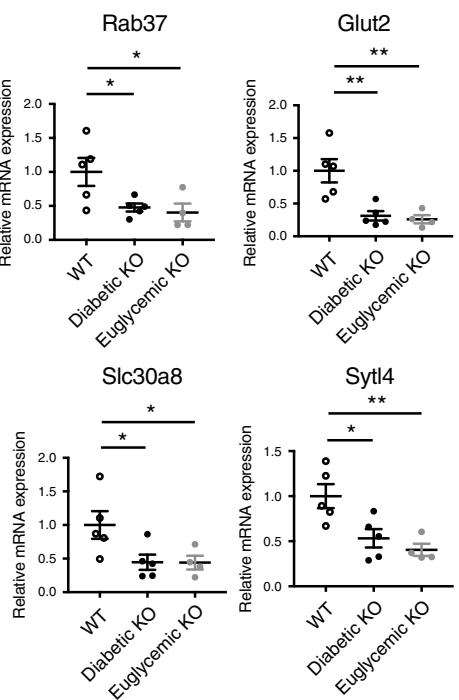

$\mathbf{f}$
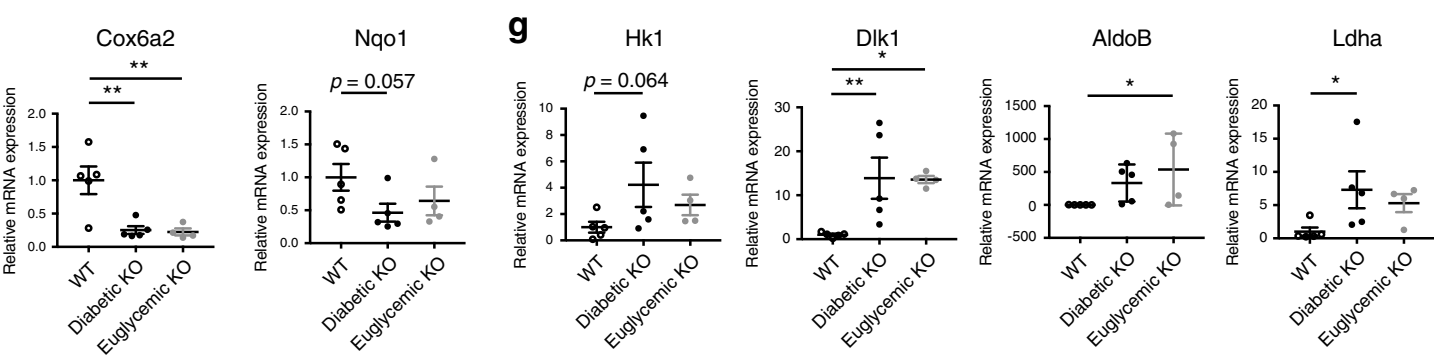

h

WT

Diabetic KO
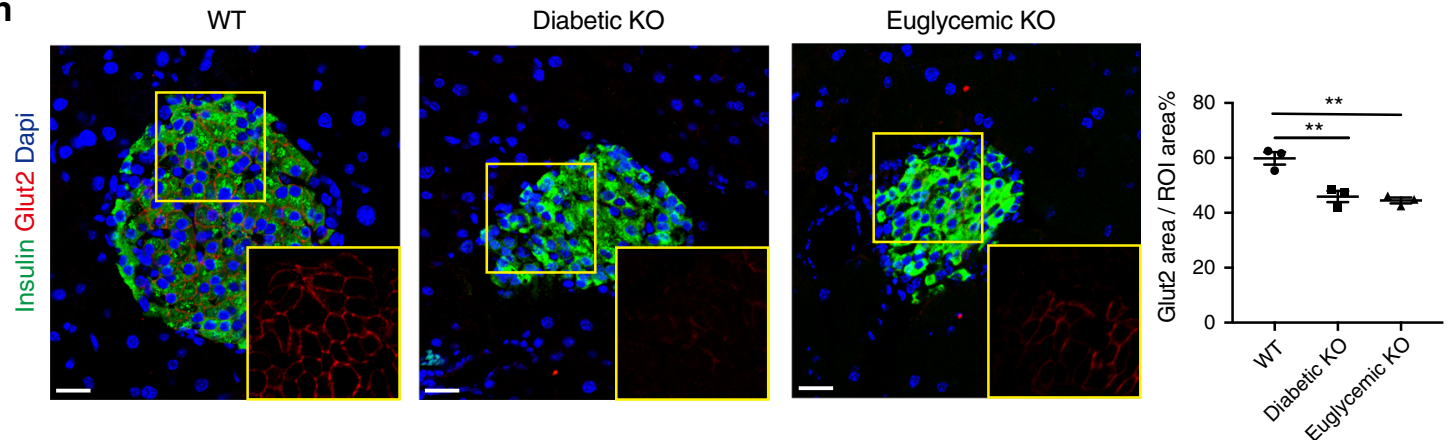

matched WT mice. At 2 weeks of age, $\beta$ RapKO ${ }^{\text {GFP }}$ mice showed normal glycemic levels (Supplementary Fig. 2a) and intact islet structure. Interestingly, we detected a dramatic induction of $M a f B$, but not $A r x$ and/or glucagon in purified mutant $\beta$-cells by qRT-PCR (Fig. 5a). We then performed immunostaining for GFP and glucagon/Arx/MafB on pancreatic sections from 2-week-old
WT and $\beta$ RapKOGFP mice. We did not detect changes in the proportion of $\mathrm{GFP}^{+} \mathrm{Gcg}^{+}$or $\mathrm{GFP}^{+} \mathrm{Arx}^{+}$cells between the two groups, but a significant increase in the percentage of $\mathrm{GFP}^{+}$cells coexpressing MafB was readily detected in mutant islets $(5.56 \% \pm$ 0.04 vs $1.99 \% \pm 0.07$ in WT, $p<0.001$ ) (Fig. $5 b, c)$. This observation was consistent with our previous finding in islet from 
Fig. 3 Impaired metabolic coupling of insulin secretion. a The number of differentially expressed genes within the three groups ( $n=3-4)$. $\mathbf{b}$ Gene expression profiles regulated by Raptor were subjected to hierarchical clustering $(n=3-4)$. c GO analysis of differentially expressed genes as identified by RNA-seq of 8-week-old WT $(n=3)$ and euglycemic $\beta$ RapKOGFP $\beta$-cells $(n=4)$ was associated with $\beta$-cell function. $\mathbf{d}$ Visualization of differential expression of genes involved in insulin secretion. $\mathbf{e - g}$ Relative expression of selected transcripts associated with $\beta$-cell secretion function $(n=5$ independent sample for WT, $n=5$ independent sample for diabetic $\beta$ RapKOGFP, $n=4$ independent sample for euglycemic $\beta$ RapKOGFP, $p$ values included in source data) (e), mitochondrial metabolism ( $n=5$ independent sample for WT, $n=5$ independent sample for diabetic $\beta$ RapKOGFP, $n=4$ independent sample for euglycemic $\beta$ RapKOGFP, $p$ values included in source data) (f), and disallowed genes $(n=5$ independent sample for WT, $n=5$ independent sample for diabetic $\beta$ RapKOGFP,$n=4$ independent sample for euglycemic $\beta$ RapKOGFP, $p$ values included in source data) (g) in WT, diabetic $\beta$ RapKOGFP, and euglycemic $\beta$ RapKOGFP $\beta$-cells by qRT-PCR. h Representative immunofluorescent staining for Glut2 (red) and insulin (green) among 8-week-old WT, diabetic $\beta$ RapKOGFP, and euglycemic $\beta$ RapKOGFP mice $(n=3)$. Insets showed different expression levels of Glut2. Quantification of Glut2 areas in Ins ${ }^{+}$ areas in WT, diabetic $\beta$ RapKOGFP, and euglycemic $\beta$ RapKOGFP mice $(n=3$, WT vs diabetic $\beta$ RapKOGFP: $p=0.002$; diabetic $\beta$ RapKOGFP vs euglycemic $\beta$ RapKOGFP: $p=0.613$; WT vs euglycemic $\beta$ RapKOGFP: $p=0.001$ ), at least 10 islets from three sections were used for quantifications of Glut2. Scale bars, $20 \mu \mathrm{m}$. Data represent means \pm SEM. ${ }^{\star} p<0.05,{ }^{\star \star} p<0.01,{ }^{\star \star \star} p<0.001$ by one-way ANOVA.

$\beta \mathrm{RapKO}^{25}$. It is known that MafB binds to and directly activates glucagon gene expression in $\alpha$-cells $s^{33}$. In order to determine whether transcriptional factor $M a f B$ can regulate glucagon expression and other $a$-cell enriched genes, we chose Glucagon ${ }^{33}$ and three other a-cell enriched genes (Corin, Fam49a, Cela1) based on MafB open chip-seq database ${ }^{34,35}$ for rescue experiments in INS-1 cells. Interestingly, SiRaptor significantly upregulated Glucagon, Corin, Fam49a, and Cela1 expression, which can be completely or partially reversed by silencing MafB expression (Fig. 5d, Supplementary Fig. 7). Taken together, Raptor is required for repressing $M a f B$ and other a-cell signature gene before the onset of hyperglycemia.

It is known that the isoform ratio of $\mathrm{C} / \mathrm{EBP} \beta$ directs cell differentiation ${ }^{36}$ and $\mathrm{mTORC} 1$ can regulate MafB expression by modulating the $\mathrm{C} / \mathrm{EBP} \beta$ isoform ratio in monocytes ${ }^{37}$. Interestingly, we found the protein level of LAP, an isoform of C/EBP $\beta$, was significantly increased in isolated islets from $\beta$ RapKOGFP mice (Fig. 5e, f). To further confirm whether mTORC1 directly regulates $\mathrm{C} / \mathrm{EBP} \beta$ and $\mathrm{MafB}$ expression, we treated INS-1 cells with mTORC1 inhibitor Rapamycin. Rapamycin treatment for 4 $\mathrm{h}$ dramatically decreased mTORC1 target PS6K expression, later at $12 \mathrm{~h}$ significantly increased C/EBP $\beta$ LAP expression and enhanced MafB promoter activity in INS-1 cells (Fig. $5 \mathrm{~g}-\mathrm{i}$ ).

We then tested whether the effect of mTORC1 on MafB expression was via $\mathrm{C} / \mathrm{EBP} \beta$. We overexpressed the $\mathrm{C} / \mathrm{EBP} \beta$ isoform LAP in INS-1 cells as a transcriptional activator (Fig. 5j), and $\mathrm{C} / \mathrm{EBP} \beta$ LAP overexpression was found to increase both MafB promoter activity (Fig. 5k) and $M a f B$ mRNA expression (Fig. 5l). The truncated C/EBP $\beta$ isoform LIP lacked the $\mathrm{N}$ terminal transactivation domains but still possessed the DNAbinding domain, thus can act as a competitive inhibitor of LAP function $^{37}$. Importantly, C/EBP $\beta$ LIP overexpression could significantly reverse Rapamycin-induced activation in MafB promoter activity and in MafB mRNA expression (Fig. $5 \mathrm{~m}, \mathrm{n}$ ). These data suggest that loss of Raptor shifts C/EBP $\beta$ to LAP isoform, which directly upregulates MafB expression and possibly contributes to multi-hormonal cell fate.

Induction of $\boldsymbol{\alpha}$-cell-enriched genes in 2-week-old $\beta$ RapKOGFP. In order to identify Raptor-dependent genes responsible for identity change, we performed transcriptomic analyses on purified $\beta$-cells sorted from 2 -week-old euglycemic $\beta$ RapKOGFP and WT mice. GO analysis revealed that the differentially expressed genes in 2-week-old $\beta$ RapKO ${ }^{\text {GFP }}$ were enriched in potassium ion transport, lactate oxidation, $\mathrm{NADH}$ oxidation, and fatty acid $\beta$ oxidation (Fig. 6a). Based on the reference lists by Qiu et al. ${ }^{31}$ and Cigliola et al. ${ }^{32}$, in 2-week-old purified GFP sorted $\beta$-cells, Raptor deficiency upregulated a subset of $\alpha$-cell-enriched genes ( 9 genes), i.e. Etv1, Fam84a, Tspan12, Spats2l, Cdo1, Adrb1, Wipf3, Pik3c2g, and Kcnk3 (Fig. 6b). We further compared the transcriptome analysis from 2- and 8-week-old datasets, and identified Raptordependent genes as those are preferentially changed at both 2and 8 -week-old euglycemic $\beta$ RapKO ${ }^{\text {GFP }} \beta$-cells (Fig. $6 \mathrm{c}$ ). This combined analysis further uncovered seven Raptor-dependent genes, including three $\beta$-cell enriched genes (Slc2a2, Msln, Ppp1r1a) and two a-cell enriched genes (Etv1, Tspan12) (Fig. 6d, e), possibly involved in $\beta$-cell identity and plasticity maintenance. We confirmed the downregulation of Slc2a2, Msln, and Ppp1r1a, and upregulation of Etv1 and Tspan12 in Raptor-deficient INS-1 cells (Fig. 6f). To determine if Etv1 and Tspan12 had direct impact on $\beta$ to a reprogramming, we overexpressed them in INS1 cells. Overexpression of Etv1 or Tspan12 alone did not result in changes in glucagon promoter activity and glucagon mRNA expression. Importantly, co-expression of Etv1 and Tspan12 caused a significant increase in both glucagon promoter activity and glucagon mRNA expression (Fig. 6g, h). Unlike some other $\mathrm{a}$ cell-enriched genes Corin, Fam49a, and Cela1, loss of Raptorinduced Etv1 and Tspan12 upregulation were not reversed by downregulation of MafB (Supplementary Fig. 7), indicating a $M a f B$-independent manner. These results suggest that Raptor directly suppress a-cell enriched genes, such as Etv1 and Tspan12 in healthy $\beta$-cells and prevent $\beta$-cell default.

\section{Discussion}

There is increasing evidence that loss of $\beta$-cell identity and the acquisition of multi-hormonal cells are occurring in the islets of patients with diabetes ${ }^{38,39}$. However, it is unclear whether these events participate in the initiation of disease or secondary to hyperglycemia during disease progression. Moreover, the pathway basis of these reprogramming events has not yet been elucidated. In the present study, we uncovered the essential role of mTORC1 in the maintenance of $\beta$-cell identity and the repression of $\alpha$-cell programs. Loss of Raptor resulted in $\beta$-cell dedifferentiation: (1) downregulation of $\beta$-cell-enriched genes, including key transcriptional factors, glucose metabolism genes, and protein secretory pathway genes; (2) the concomitant upregulation of $\beta$ cell disallowed genes; and (3) the likely upregulation of $\beta$-cell progenitor markers, i.e. Ngn3, Aldh1a3, Sox9. Moreover, loss of Raptor resulted in the appearance of $\alpha$-cell features in lineagelabeled $\mathrm{GFP}^{+} \beta$-cells, manifested by gaining the expressions of glucagon and $\alpha$-cell key transcription factors MafB/Arx, displaying both $\beta$ - and $\alpha$-like secretory granules and induction of a group of $\alpha$-cell enriched genes. Importantly, when 4 -week-old Raptor-deficient mice were maintained at normoglycemia for 4 weeks, the euglycemic mutant $\mathrm{GFP}^{+} \beta$-cells still exhibited downregulation of $N k x 6.1, P d x 1$, and Glut2, upregulation of progenitor markers and a group of $\alpha$-cell enriched genes, as well as ectopic expression of dedifferentiation marker ALDH1A3. There remained 13-fold increase in $\mathrm{Gcg}^{+} \mathrm{GFP}^{+}$multi-hormonal cells in euglycemic mutants compared to WT, whereas 
a

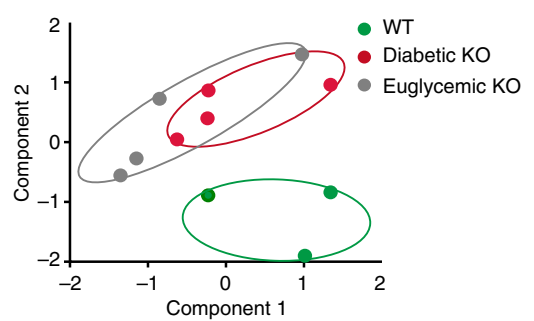

b

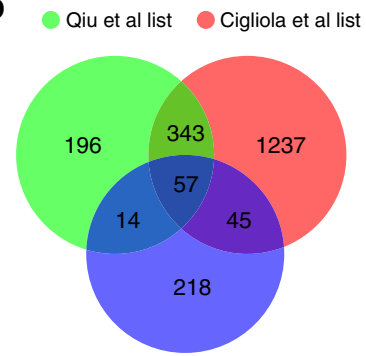

d

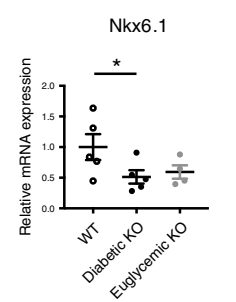

Gcg

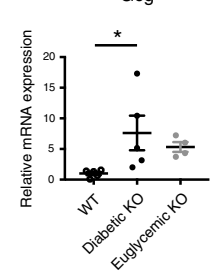

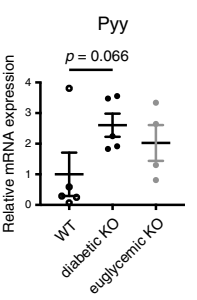

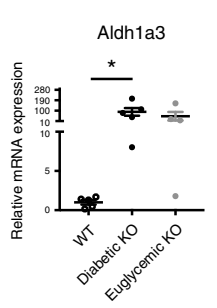

$\operatorname{Arx}$
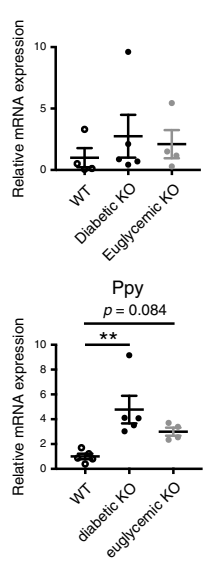
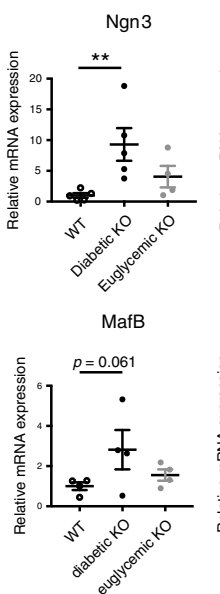

Slc38a5
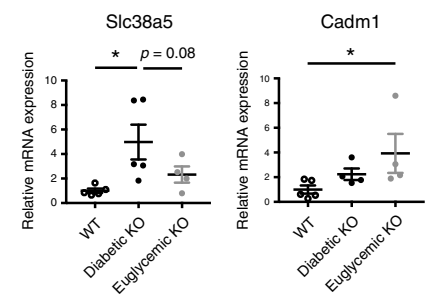

C

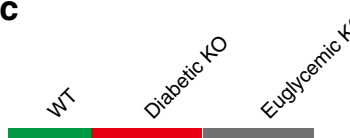

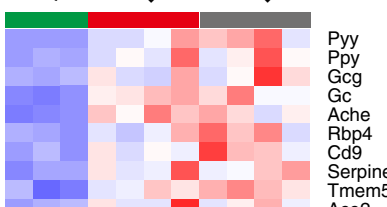

mem591

Ace2

Pou6f2

Sorcs2

Etv1

Grra1

Cav2

Oxtr

Mapk4

Mapk4
Pappa2

Ehf

Ptprk

Rria2
Rasgrf2
Zfp36l1

Zorin

Adamts 18
Wfdc16

Gpx3

Tfpi

Rhou
Smarca1
C8b

Sema3e

Sema3

Slc2a3

Rab27b

Tspan12

Arx
Fam49a
Arg1

Arg1
Thsd7a

Thsd7a

Trf

Ocrl

Clc38a5

Slc38a5

Cadm1
Emp1
Krtap17-1

Krtap17-1

Sh3bgrl2

Id 1

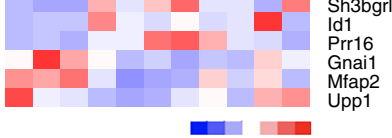

e
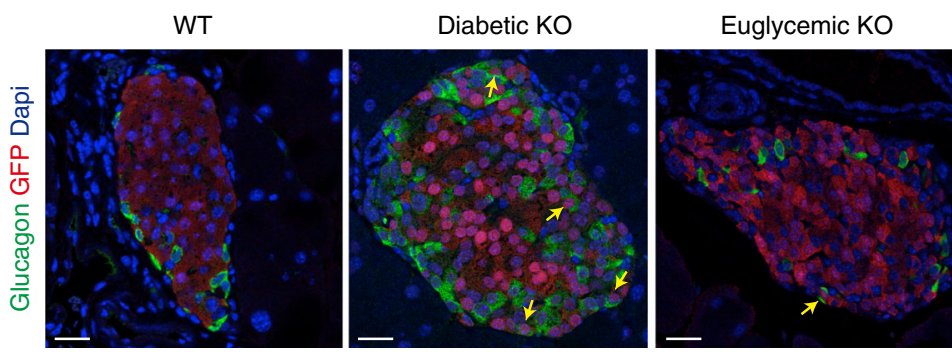

f
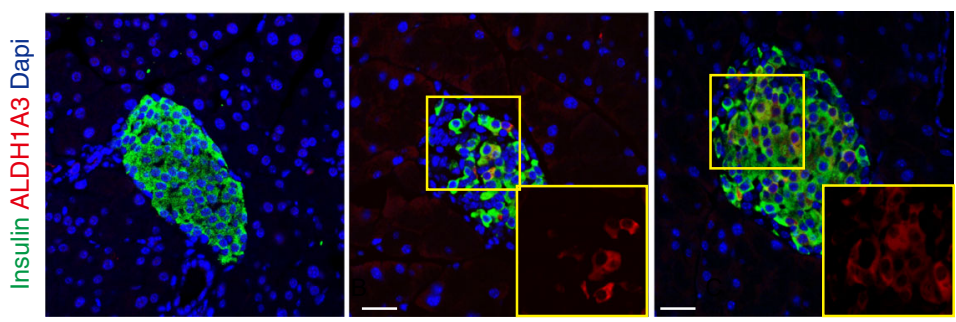
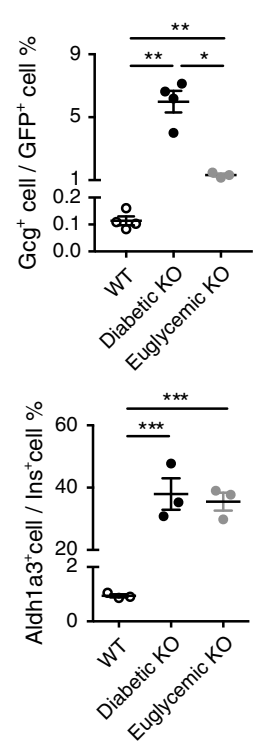

hyperglycemia indeed further propelled the reprograming process. These data demonstrate that in the absence of mTORC1, loss of $\beta$-cell identity and the acquisition of non- $\beta$ cell program could not be completely reversible upon normalization of glucotoxicity.

Interestingly, in 2 -week-old euglycemic $\beta$ RapKOGFP $\beta$-cell, $\alpha$ cell critical transcription factor $M a f B$ and a group of $\alpha$-cell- enriched genes were already strongly induced. We provided evidence that $\mathrm{mTORC1}$ regulated $\mathrm{MafB}$ promotor activity and expression via modulating the $\mathrm{C} / \mathrm{EBP} \beta$ isoform ratio of LAP and $\operatorname{LIP}^{37,40}$, thus participated in $\beta$ to $\alpha$ reprogramming. Our data were consistent with a previous observation that ectopic expression of LAP and LIP in monocytes differentially activated MafB expression and osteoclast-genesis ${ }^{41}$. MafB has been shown to 
Fig. 4 Induction of $\boldsymbol{\alpha}$-cell-enriched genes in euglycemic $\boldsymbol{\beta}$ RapKOGFP. a $\alpha$-Cell-enriched genes and $\beta$-cell-enriched genes were subjected to perform principle component analysis based on the dataset from Qiu et al. ${ }^{31}$. b Venn diagram representation of the subsets of Raptor-regulated genes that were enriched in $\alpha$-cells. c Heatmap showed the differential expression of 57 overlapped genes in 8-week-old WT, diabetic $\beta$ RapKOGFP, and euglycemic $\beta$ RapKOGFP $\beta$-cells $(n=3-4)$. d Relative expression of genes involved in cell identity by qRT-PCR $(n=5$ independent sample for WT, $n=5$ independent sample for diabetic $\beta$ RapKOGFP, $n=4$ independent sample for euglycemic $\beta$ RapKO GFP, $p$ values included in source data). e Representative immunofluorescent staining for GFP (red) and glucagon (green) among 8-week-old WT, diabetic $\beta$ RapKOGFP, and euglycemic $\beta$ RapKOGFP mice ( $n=4$ for WT, $n=4$ for diabetic $\beta$ RapKOGFP, $n=3$ for euglycemic $\beta$ RapKOGFP). The yellow arrows indicated glucagon and GFP co-stained cells. Percentage of $\mathrm{Gcg}^{+} \mathrm{GFP}^{+}$cells among GFP+ cells in WT, diabetic $\beta$ RapKOGFP, and euglycemic $\beta$ RapKO GFP mice was determined $(n=4$ independent sample for WT, $n=$ 4 independent sample for diabetic $\beta$ RapKOGFP, $n=3$ independent sample for euglycemic $\beta$ RapKOGFP, WT vs diabetic $\beta$ RapKOGFP: $p=0.007$; diabetic $\beta$ RapKOGFP vs euglycemic $\beta$ RapKOGFP: $p=0.012$; WT vs euglycemic $\beta$ RapKOGFP: $p=0.009$ ). At least 50 islets were used for quantifications. Scale bars, $20 \mu \mathrm{m}$. f Representative immunofluorescent staining for ALDH1A3 (red) and insulin (green) among 8-week-old WT, diabetic $\beta$ RapKOGFP, and euglycemic $\beta$ RapKOGFP mice $(n=3)$. Insets showed different expression levels of ALDH1A3. Percentage of ALDH1A3 ${ }^{+}$Ins ${ }^{+}$cells among Ins ${ }^{+}$cells in WT, diabetic $\beta$ RapKOGFP, and euglycemic $\beta$ RapKOGFP mice was calculated ( $n=3$, WT vs diabetic $\beta$ RapKOGFP: $p<0.001$; diabetic $\beta$ RapKOGFP vs euglycemic $\beta$ RapKOGFP: $p=0.628$; WT vs euglycemic $\beta$ RapKOGFP: $p<0.001$ ). For ALDH1A3 quantification, at least 36 islets were used. Scale bars, $20 \mu \mathrm{m}$. Data represent means \pm SEM. ${ }^{\star} p<0.05,{ }^{\star \star} p<0.01,{ }^{\star \star \star} p<0.001$ by one-way ANOVA.

activate cell type-specific expression of the glucagon gene ${ }^{33}$. In the present study, we found Raptor/C/EBP $\beta /$ MafB not only regulates glucagon mRNA expression but also controls the transcription levels of some a-cell-enriched genes, i.e. Corin, Cela1, and Fam49a. A further combined analysis on 2- and 8-week-old transcriptome revealed seven genes that were Raptor specifically dependent, including a-cell enriched genes Etv1 and Tspan12. Etv1 belongs to the Pea3 group of the ETS family of transcription factors ${ }^{42-44}$ and Tspan12 is a known component of Tetraspanin (Tspan12) family ${ }^{45-}$ 47; however, their functions in islet $\beta$-cell and $\alpha$-cell are still unknown. Our data showed that co-expression of Etv1 and Tspan12 caused a significant increase in glucagon promoter activity and glucagon mRNA expression in $\beta$-cells. This is relevant since $\beta$-cellspecific ETV-dependent genes was significant enrichment of diabetes and obesity-associated genes, thus involving the pathogenesis of diabetes ${ }^{48}$. To our knowledge, this is the first time a metabolic pathway has been identified not only maintaining the proper expression of $\beta$-cell functional genes but also repressing non- $\beta$ cell genes. Conceptually similar findings were reported for $P d x 1$ (prevent glucagon) ${ }^{6}, N k x 6.1$ (prevent somatostatin) ${ }^{7}, N k \times 2.2$ (prevent somatostatin) ${ }^{9}$, and Dnmt1 (prevent glucagon) ${ }^{49}$ in preventing acquisition of non- $\beta$-cell hormones.

Previous studies have illustrated that metabolic defect predisposes to $\beta$-cell dedifferentiation and reprogramming ${ }^{2,50}$. Failure $\beta$-cells show conjoined features of two cardinal processes: mitochondrial dysfunction and progenitor-line features ${ }^{5}$. Raptordeficient dedifferentiated $\beta$-cell was characterized by low UCN3, low GLUT2, and high ALDH1A3 expression, with destabilized $\beta$ cell metabolic coupling of insulin secretion. Euglycemic Raptordeficient $\beta$-cell exhibited downregulation of mitochondria genes (Nqo1, Atp4a, mt-Co3, mt-Nd2, mt-Co1, and Cox6a2), decreased Glut2 expression, derepressed disallowed genes (HK1 (ref. ${ }^{51}$ ), $A l d o B^{52}$, and $L d h a^{53}$ ) and reduced ATP levels for GSIS. It has been reported that mTORC1 controls mitochondrial dynamics, possibly due to the preferential translational regulation of a subset of cellular mRNA for essential nucleus-encoded mitochondrial proteins $^{54,55}$. The mitochondrial defect in Raptor-deficient $\beta$-cell was reminiscent of dedifferentiated $\mathrm{ALDH}^{+} \beta$-cells, in which mitochondrial complex I, IV, and V function were significantly impaired. It is logical that impaired mitochondrial complex I, IV, and $\mathrm{V}$ functions can lead to reduced ATP production, stalling of protein translation and reactivation of genes that sustain a cellular progenitor program ${ }^{56}$. We propose that impaired mitochondrial function in Raptor-deficient $\beta$-cells marks the progression from mitochondria defect to $\beta$-cell identity loss and reprogramming in the natural history of $\beta$-cell failure before diabetes occurs.

In addition to mitochondria metabolic uncoupling, loss of Raptor directly impaired proinsulin synthesis, the most energy consuming processes in $\beta$-cell. This was evidenced by dramatic reduction in proinsulin content in euglycemic mutant pancreas and islet, when C-peptide was completely reversed after glucose normalization. On the contrary, the proinsulin/insulin ratio "flaws" detected in diabetic $\beta$ RapKOGFP by us and BlandinoRosano et al. ${ }^{26}$ disappeared when mutant mice were constantly kept at normoglycemia. The decreased expression of CPE, a pancreatic convertase, in diabetic $\beta$ RapKOGFP mice ${ }^{26}$, was reversed in euglycemic $\beta$ RapKO GFP mice. In diabetes, $\beta$-cells undergo marked degranulation of mature secretory granule (SG) in response to hyperglycemia, which stimulates secretion. This leads to decreased pancreatic C-peptide and CPE, which also resides in SG. This is found in $\beta$ RapKOGFP mice, which are insulin deficient to begin with, as well as in other models of diabetes ${ }^{57,58}$. Treatment with insulin prevents hyperglycemia and restores the SG pool and consequently increases insulin (C-peptide) content, along with reduced proinsulin/C-peptide ratio. Increased proinsulin/C-peptide ratio per se may represent accumulation of young SG, as the mature granules are being secreted; whether it also imply the processing defects needs to be confirmed by pulse-chase experiments. Likewise, genes involved in ROS production and anti-inflammatory process were also recovered after glycemic normalization. This is consistent with the finding that chronic hyperglycemia is highly associated with upregulation of inflammatory mediators in both animals and in human diabetic subjects ${ }^{59}$, while insulin can ameliorate the inflammatory responses ${ }^{60}$. Moreover, hyperglycemia increased ROS production by driving mitochondria toward increased oxygen use and decreased ATP formation ${ }^{61,62}$, which in turn suppressed insulin generation in pancreatic $\beta$-cells by inhibiting insulin gene transcription factors such as Pdxl or MafA ${ }^{63,64}$. Taken together, our work identified the importance of mTORC1 signaling for metabolic coupling and proinsulin synthesis in $\beta$-cells, while attributed the inappropriate proinsulin/insulin ratio, ROS, and inflammatory responses in $\beta$ RapKOGFP to glucotoxicity.

In light of the current data, we propose Raptor controls $\beta$-cell anabolic metabolism and serves as checkpoint of $\beta$-cell identity and plasticity (Fig. 7). At euglycemia, loss of Raptor results in $\beta$ cell dedifferentiation (marked as UCN3 $3^{\text {low }}$, GLUT2 $2^{\text {low, and }}$ ALDH1A3 ${ }^{\text {high }}$ ) with compromised $\beta$-cell identity, metabolic uncoupling, and regression to multi-hormonal state with $\alpha$-cell features. We propose Raptor/C/EBP $\beta / M a f B$-dependent and $M a f B$-independent ways in silencing $\alpha$-cell-enriched genes and glucagon expression in healthy $\beta$-cells. Raptor-deficient $\beta$-cells with compromised identity become dysfunctional and eventually cause diabetes; in turn, hyperglycemia further aggravates dedifferentiation and reprogramming process. Our data provide insights into the molecular nature of dedifferentiation process that independent of metabolic state, and by translating mTORC1$\beta$ cell default biology into mechanism-based interventions might help to reverse the course of diabetes. 
a

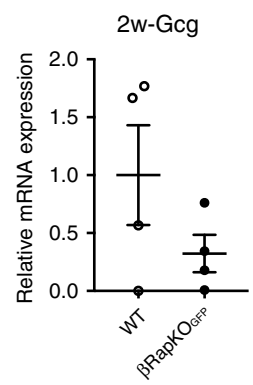

b
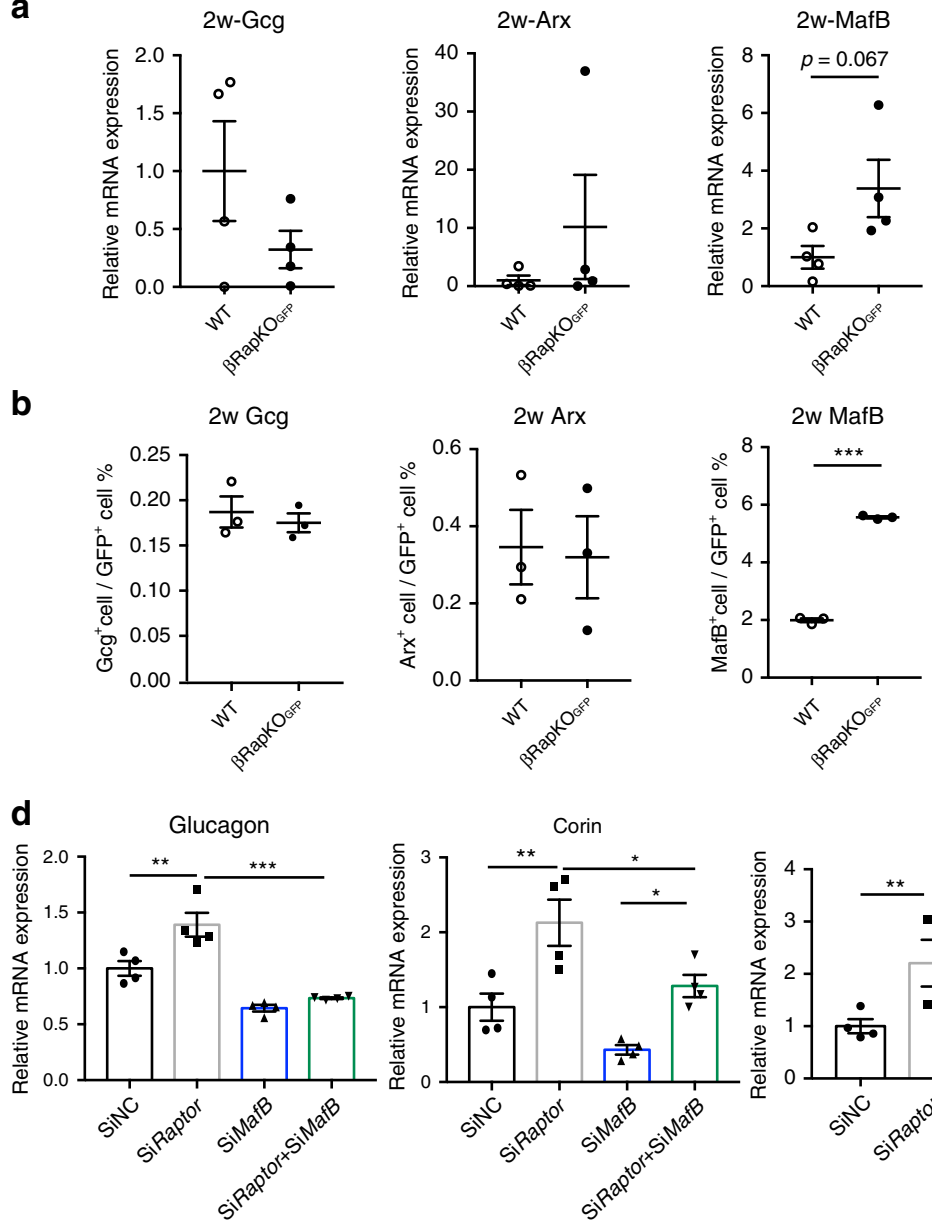

c

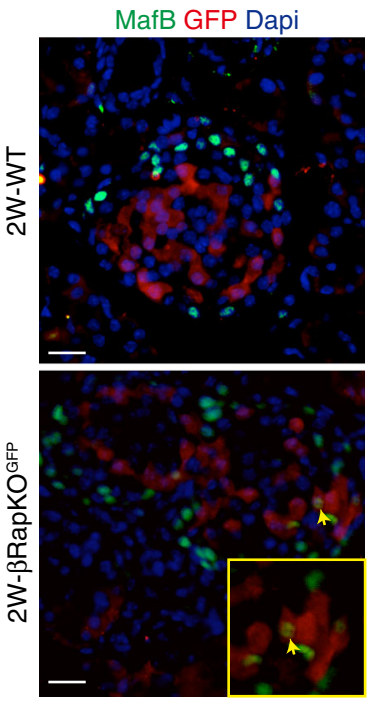

e

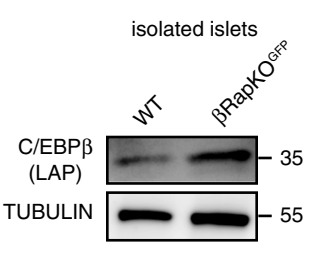

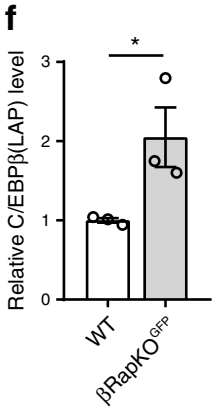

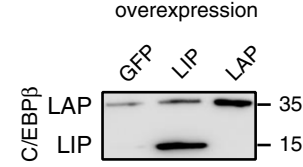

TUBULIN

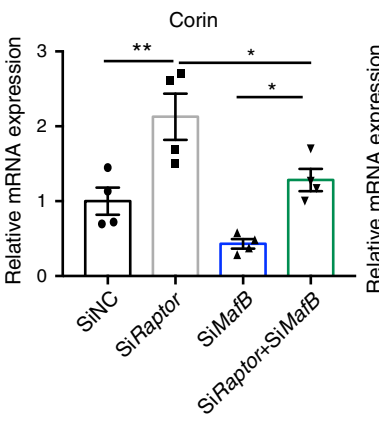

9

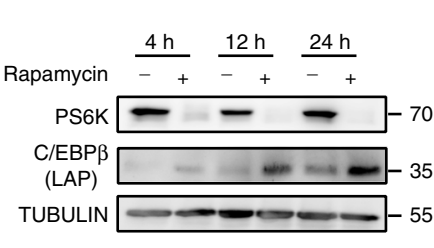

Fam49a

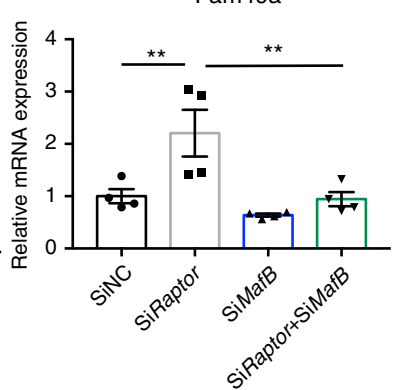

h

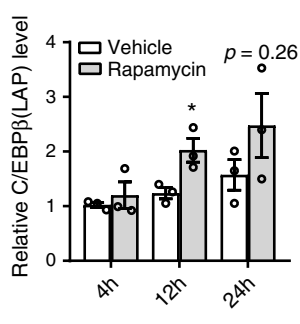

k

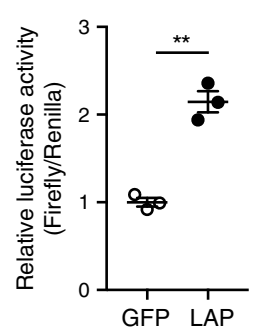

1

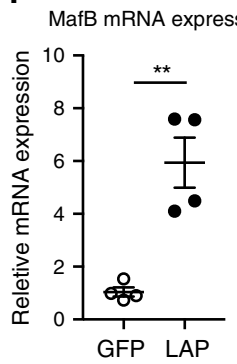

m

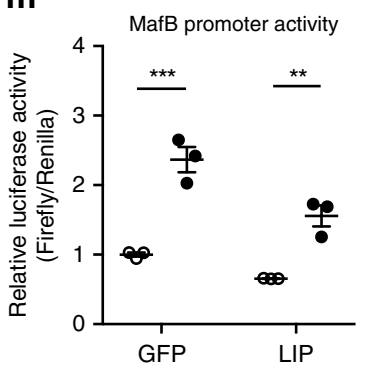

Cela1

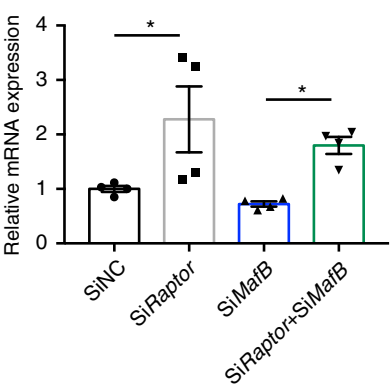

i MafB promoter activity

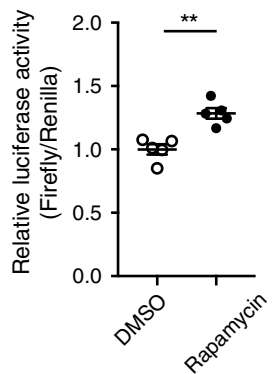

n

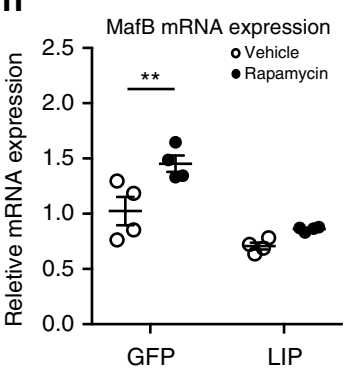

\section{Methods}

Mice and supplementation with insulin using osmotic pumps. $\beta$-Cell-specific Raptor knockout mice ( $\beta$ RapKO) were generated by crossing Raptorflox/flox

mice (purchased from the Jackson Laboratory, C57BL/6J) with mice expressing Cre recombinase driven by the rat insulin promoter (RIP-Cre, mixed C57BL/ 6J:129/SvJ) and were further bred to Rosa26-EGFP mice (purchased from the Jackson Laboratory, USA) to generate $\beta$ RapKO GFP mice (Raptor ${ }^{-1-}$, RIP-Cre,
Rosa $26^{G F P}$ ), permitting lineage tracing of Raptor-deleted cells. $\beta$ RapKOGFP mice were used for experiments and their age-matched littermates (Raptor ${ }^{+/+}$, RIP-Cre, Rosa26 ${ }^{G F P}$ ) were used as WT controls. Genotyping was performed by PCR using genomic DNA isolated from the toe tips of newborn mice. Only male animals were used for the experiments. Animals were maintained in a specific pathogen-free animal facility with $22 \pm 1{ }^{\circ} \mathrm{C}, 60-70 \%$ humidity on a 12 -h

light-dark cycle. Water and food were provided ad lib. All animal experiments 
Fig. 5 mTORC1 regulates $\alpha$-cell transcriptional factor MafB. a Relative expression of glucagon, Arx, and MafB in 2-week-old WT and $\beta$ RapKOGFP $\beta$-cells by qRT-PCR $(n=4)$. b Quantification of Gcg ${ }^{+} \mathrm{GFP}^{+}, \mathrm{Arx}^{+} \mathrm{GFP}^{+}$, and MafB ${ }^{+} \mathrm{GFP}+$ cells in GFP ${ }^{+}$cells in 2-week-old WT and $\beta$ RapKO GFP mice $(n=3, \mathrm{Gcg}: p=$ 0.58; Arx: $p=0.86$; MafB: $p=9.00122 \mathrm{E}-07$ ). At least $1783 \mathrm{GFP}+$ cells were used for quantifications. $c$ Representative immunofluorescent staining for MafB (green) and GFP (red) in 2-week-old WT and $\beta$ RapKOGFP mice $(n=3)$. Yellow box showed the specific area of the islet, which was enlarged and represented by arrows to demonstrate $\mathrm{MafB}^{+} \mathrm{GFP}^{+}$cell. Scale bars, $20 \mu \mathrm{m}$. d Relative expression of selected transcripts associated with $\alpha$-cell-enriched genes in INS-1 cells which were transfected with SiNC or SiRaptor in the presence or absence of SiMafB by qRT-PCR ( $n=4$ independent cell experiments, $p$ values included in source data). e, $\mathbf{f}$ Western blotting showed the expression of C/EBP $\beta(\mathrm{LAP})$ in 8-week-old WT and $\beta$ RapKOGFP mice $(n=3, p=0.049)$. $\mathbf{g}$, $\mathbf{h}$ INS-1 cells were treated with Rapamycin ( $25 \mathrm{nM}), \mathrm{C} / \mathrm{EBP} \beta(\mathrm{LAP})$ protein expression was assayed by immunoblot ( $n=3$ independent cell experiments). i Luciferase reporter gene assays revealed that Rapamycin treatment for $12 \mathrm{~h}$ positively regulated the luciferase activity of MafB ( $n=5$ independent cell experiments, $p=0.0011)$. $\mathbf{j}$ Immunoblotting to evaluate the LAP and LIP protein levels after LAP and LIP overexpression ( $n=2$ independent cell experiments). $\mathbf{k}$ Luciferase reporter assay using a rat MafB promoter reporter. INS-1 cells were transfected with GFP or LAP overexpression vector ( $n=3$ independent cell experiments, $p=0.001$ ). I qRT-PCR analysis of MafB expression in GFP and LAP overexpressed INS-1 cells ( $n=4$ independent cell experiments, $p=0.0023$ ). $\mathbf{m}$ Luciferase reporter assay using a rat MafB promoter reporter. INS-1 cells were transfected with GFP or LIP overexpression vector in the absence and presence of Rapamycin ( $n=3$ independent cell experiments, GFP + Vehicle vs GFP + Rapamycin, $p<0.001 ;$ LIP + Vehicle vs LIP + Rapamycin, $p=0.001)$. $\mathbf{n}$ qRT-PCR analysis of MafB expression in GFP and LIP overexpressed INS-1 cells in the absence or presence of Rapamycin ( $n=4$ independent cell experiments, GFP + Vehicle vs GFP + Rapamycin, $p=0.002$; LIP + Vehicle vs LIP + Rapamycin, $p=0.177$ ). Data represent means \pm SEM ${ }^{\star} p<0.05,{ }^{\star \star} p<0.01,{ }^{\star \star \star} p<0.001$ by two-sided Student's $t$-test and one-way ANOVA.

were approved by the Animal Care Committee of Shanghai Jiao Tong University School of Medicine.

Four weeks after birth, some $\beta$ RapKOGFP mice were anesthetized by intraperitoneal injection of $1 \%$ Pelltobarbitalum Natricum and subcutaneously implanted with Alzet mini-osmotic pumps (model 1004; Charles River Laboratories, USA) at a dose of $0.2-0.3 \mathrm{U} /$ day over a period of 28 days supplementing with human insulin (Novolin ${ }^{\oplus} \mathrm{R}$ Penfill ${ }^{\mathbf{\Phi}}$, Novo Nordisk, Denmark). Blood glucose was measured every other day over the entire period of the experiment. Twenty-eight days after pump implantation, mice were sacrificed and pancreas was removed.

Metabolic studies. Blood glucose was monitored every 2 weeks. We performed glucose tolerance tests on overnight-fasted mice by intraperitoneal injection of $2 \mathrm{~g} /$ $\mathrm{kg}$ glucose. Blood glucose concentration was measured before and 15, 30,60, and 120 min after glucose injection by glucometers (Accu-Chek, Roche, Mannheim, Germany). Six-hour-fasting serum insulin concentration was measured by ELISA Kit (Mouse Ultrasensitive Insulin ELISA Kit, Alpco, USA) and $6 \mathrm{~h}$-fasting serum glucagon concentration was measured by radioimmunoassay kit (Glucagon ELISA Kit, Chemiluminescent, Millipore, USA).

Pancreas was removed and disrupted using ethanol-HCl extraction and homogenizer (Fisher Scientific, USA). Samples were centrifuged, and the supernatant after centrifugation was used for measurement of total C-peptide/ proinsulin content per pancreas. The C-peptide and proinsulin content were analyzed by C-peptide (Mouse C-Peptide ELISA Kit, Crystal Chem, USA) and proinsulin (Rat/Mouse Proinsulin ELISA, Mercodia, Sweden) ELISA kits and normalized to pancreas protein concentration (BCA method; ThermoFisher Scientific, USA)

$\boldsymbol{\alpha}$-Cell mass and $\boldsymbol{\beta}$-cell mass measurement. Whole pancreas from 8 -week-old mice were weighed, fixed, embedded, and sectioned continuously. At least 10 evenly $200 \mu \mathrm{m}$ apart sections throughout the entire pancreas were picked to immunostaining for insulin, glucagon, followed by peroxidase conjugated secondary antibody, visualized using a DAB Peroxidase Substrate Kit (Fuzhou Maixin Biotech, China), and counterstained with eosin ${ }^{65}$. Digital images of whole pancreas were captured by a Nikon MZ 100 microscope (Japan). Total pancreatic and insulin/glucagon positive areas of each section were measured and calculated using Meta-Morph version6.1 (Molecular Devices, USA). $\beta$-Cell mass/ $\alpha$-cell mass was obtained by multiplying the ratio of total insulin-positive area (total glucagon positive area) to total pancreatic area with the pancreas weight.

Immunofluorescence. Pancreas tissues were fixed in $4 \%$ paraformaldehyde overnight at $4{ }^{\circ} \mathrm{C}$, embedded in paraffin, and cut into $6-\mu \mathrm{m}$-thick section. For nuclear transcription factor detection, we performed antigen retrieval in citrate buffer (Vector Laboratories, USA) for $15 \mathrm{~min}$ in the microwave. Slides were washed with PBS and blocked with normal donkey serum (5\%), and then primary antibodies were applied (Supplementary Table 1).The Arx antibody was from Dr.Kunio Kitamura's lab66. For specific antibodies, biotin-streptavidin system (Fuzhou Maixin Biotech, China) and Tyramide signal amplification (PerkinElmer, USA) were performed. Fluorescent secondary antibodies were from Invitrogen (USA) (Supplementary Table 2). Slides were mounted with Vectashield with DAPI (SouthernBiotech, USA). We used confocal microscopy (Zeiss710, Germany) and Laser Scanning Microscope Software (Zen (blue edition)/2.1) to survey colocalization and capture images. Immunofluorescent images were analyzed by ImageJ software (edition/1.50i, NIH).
Isolated islets were dispersed into single cells with $0.25 \%$ trypsin-EDTA (Gibco, USA), then cells were cultured on a microscope slide (Millipore's Millicell EZ SLIDE, USA) overnight, washed with PBS and fixed in $4 \%$ pre-cooling paraformaldehyde for $20 \mathrm{~min}$, and then the fixed cells were stained for PS6 and insulin.

Isolation of pancreatic islets and purification of $\boldsymbol{\beta}$-cells. Pancreatic islets were isolated by collagenase $\mathrm{P}$ (Roche, Switzerland) injecting into the common bile duct. The perfused pancreas was dissected and incubated at $37^{\circ} \mathrm{C}$ for $17 \mathrm{~min}$. Digested exocrine cells and intact islets were separated via centrifugation, and intact islets were manually handpicked.

Islets were digested into single cells by $0.25 \%$ trypsin-EDTA (Gibco, USA) solution and sorted for GFP by FACS (MoFlo XDP, Beckman Coulter, USA). Cells from Raptor ${ }^{+/+}$Rosa $26^{G F P}$ mice were used as a negative control for FACS gating. $\beta$-Cell-derived endocrine cells lineage traced with GFP were purified by FACS sorting to a purity of $85-95 \%$. Sorted cells were then processed for RNA extraction.

RNA-sequencing analysis. Total RNA was isolated using RNeasy Micro kit (Qiagen, Germany). Paired-end libraries were synthesized by using the TruSeq ${ }^{\oplus}$ RNA Sample Preparation Kit (Illumina, USA) following TruSeq ${ }^{\oplus}$ RNA Sample Preparation Guide. Briefly, the poly-A-containing mRNA molecules were purified using poly-T oligo-attached magnetic beads. Following purification, the mRNA is fragmented into small pieces using divalent cations under $94^{\circ} \mathrm{C}$ for $8 \mathrm{~min}$. The cleaved RNA fragments are copied into first-strand cDNA using reverse transcriptase and random primers. This is followed by second-strand cDNA synthesis using DNA Polymerase I and RNase H. These cDNA fragments then go through an end repair process, the addition of a single "A" base, and then ligation of the adapters. The products are then purified and enriched with PCR to create the final cDNA library. Purified libraries were quantified by Qubit ${ }^{\oplus} 2.0$ Fluorometer (Life Technologies, USA) and validated by an Agilent 2100 bioanalyzer (Agilent Technologies, USA) to confirm the insert size and calculate the mole concentration. Cluster was generated by cBot with the library diluted to $10 \mathrm{pM}$ and then were sequenced on the Illumina HiSeq 2500 (Illumina, USA). The library construction and sequencing was performed at Shanghai Biotechnology Corporation. Sequencing raw reads were preprocessed by filtering out rRNA reads, sequencing adapters, short-fragment reads, and other low-quality reads. We used Tophat v2.1.0 (ref. ${ }^{67}$ ) to map the cleaned reads to the mouse mm10 reference genome with two mismatches. After genome mapping, Cufflinks v2.1.1 (ref. ${ }^{68}$ ) was run with a reference annotation to generate FPKM values for known gene models. Differentially expressed genes were identified using Cuffdiff ${ }^{68}$. The $p$ value significance threshold in multiple tests was set by the false discovery rate (FDR). The fold-changes were also estimated according to the FPKM in each sample. The differentially expressed genes were selected using the following filter criteria: FDR $\leq 0.05$ and fold change $\geq 1.5$.

Microarray analysis. Total RNA was extracted from freshly purified $\beta$-cells of 2 week-old $\beta$ RapKOGFP and WT mice using RNeasy Micro Kit (Qiagen, Germany) and sample qualities were assessed using an Agilent Bioanalyzer 2100 (Agilent technologies, Santa Clara, CA, USA), taking a minimal cut-off RIN $\geq 7$. We included at least 3-4 independent biological replicates, each composed of RNA isolated from $\beta$-cells of 5-6 animals with the same genotype. Total RNA samples of each group were then used to generate biotinylated cRNA targets for the Affymetrix Gene Clariom S Array. Data were analyzed using Genespring software (Agilent). 
a

\begin{tabular}{ll}
$\begin{array}{ll}\text { 2w-WT vs } 2 w-\beta R a p K O \\
\text { GO Biological Function }\end{array}$ & $P$ value \\
\hline Potassium ion import across plasma membrane & 0.000293 \\
\hline Potassium-transporting ATPase activity & 0.000865 \\
\hline Cellular response to increased oxygen levels & 0.001072 \\
\hline Lactate oxidation & 0.001409 \\
\hline Glycogen catabolic process & 0.002958 \\
\hline Fatty acid $\beta$ oxidation using acyl-CoA oxidase & 0.003471 \\
\hline NADH oxidation & 0.004824 \\
\hline Oxygen metabolic process & 0.004824 \\
\hline
\end{tabular}

b
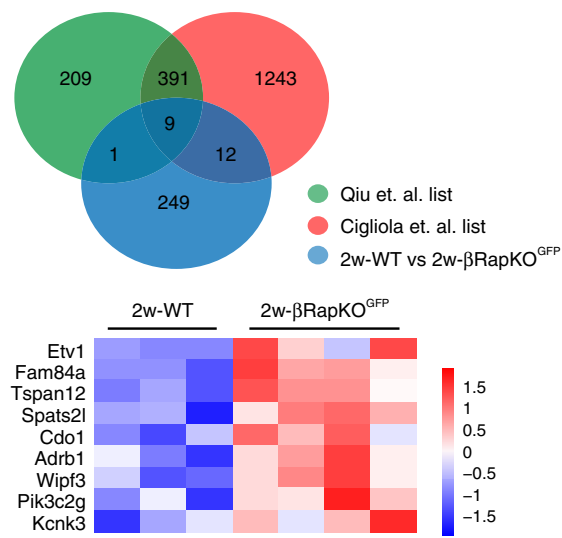

C

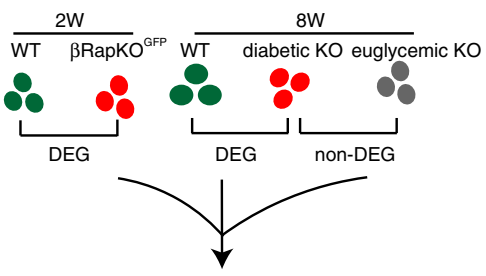

Raptor dependent genes

e

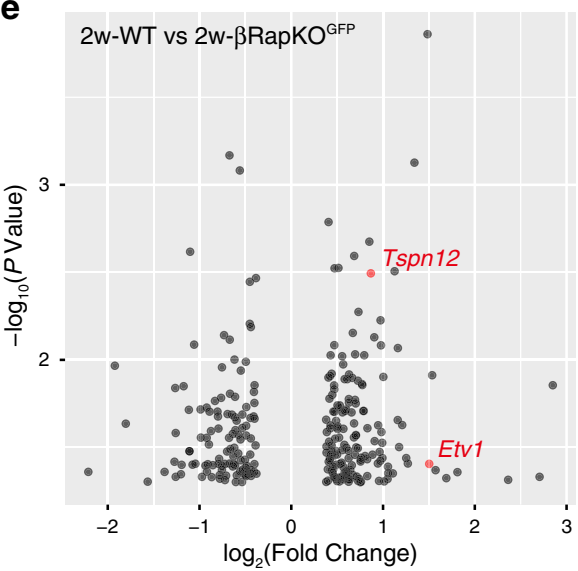

g

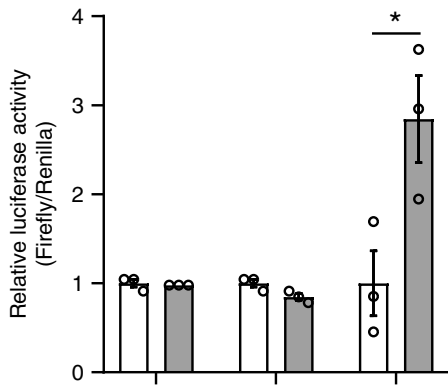

Overexpression

$\begin{array}{rllllll}\text { Etv1 } & - & + & - & - & - & + \\ \text { span12 } & - & - & - & + & - & +\end{array}$

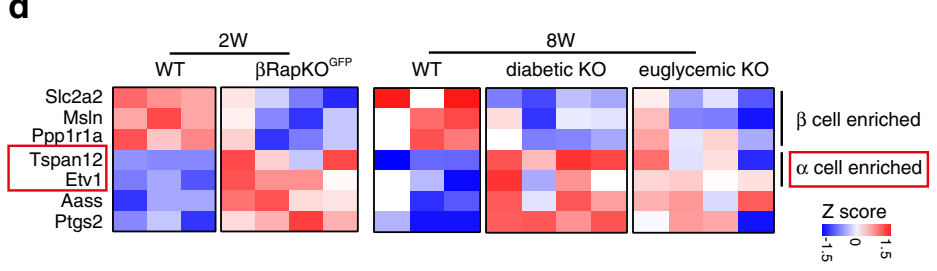

f

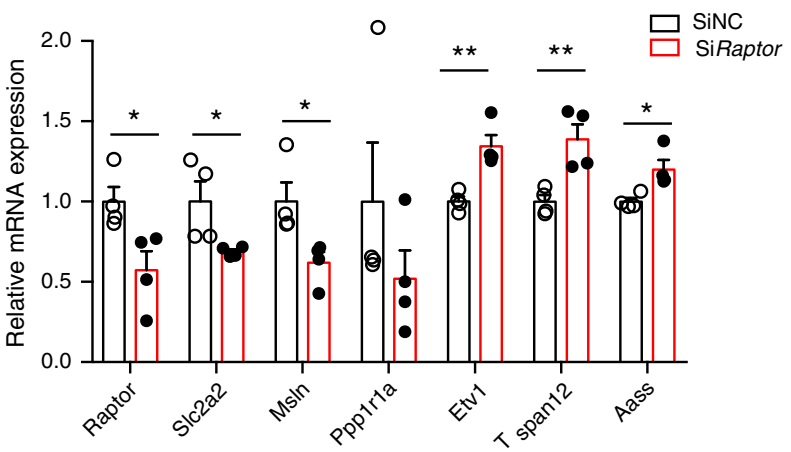

h

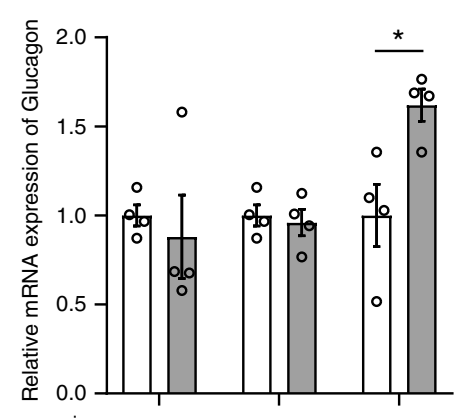

Overexpression

Etv1 - + - $-\quad-+$

Tspan12 - $-\quad$ - $\quad$ - +
qRT-PCR analysis and western blotting. For qRT-PCR, total RNA was extracted by TRIzol reagent (Invitrogen, USA) according to the manufacturer's protocols. One microgram of RNA was used for synthesis of cDNA using SuperScriptII (Invitrogen, USA) with random primer as described. Duplicate samples for quantitative PCR were run in an ICycler (ABI, California, USA, QuantStudio 12K Flex Software/v1.2.2). $\Delta$ Ct for each gene was determined after normalization to actin, and $\Delta \Delta \mathrm{Ct}$ was calculated relative to the control. Gene expression values were then expressed as a fold change, calculated by $2^{-\Delta \Delta C t}$. Primer sequences can be obtained in Supplementary Table 3.

The protein concentration of islet and cell lysates was quantified by BCA protein assay (Pierce, Rockford, IL, USA). Protein samples $(20 \mu \mathrm{g})$ were separated by electrophoresis on TGX precast gel 4-20\% (Bio-Rad, Hercules, CA, USA) and transferred to a polyvinylidene fluoride (PVDF) membrane. Primary antibodies are listed as follows: rabbit anti-RAPTOR (1:1000; Cell Signaling Technology, USA), rabbit anti-PS6 (Ser240/244) (1:1000; Cell Signaling Technology, USA), rabbit anti4E-BP1 (1:1000; Cell Signaling Technology, USA), rabbit anti-tubulin (1:1000; Cell Signaling Technology, USA), rabbit anti-CPE (1:1000; GeneTex, USA), and guinea pig polyclonal anti-insulin antibody from Liuming Lab ${ }^{69}$. TUBULIN was used as an internal control to normalized band intensity. Horseradish peroxidase-coupled goat-anti-rabbit IgG (1:2000; Cell Signaling Technology, USA) and horseradish peroxidase-coupled goat-anti-guinea pig IgG (1:2000; Jackson ImmunoResearch, USA) were used as a secondary antibody. Blots were developed with enhanced 


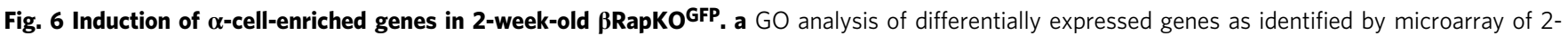
week-old WT $(n=3)$ and $\beta$ RapKOGFP $\beta$-cells $(n=4)$ was associated with $\beta$-cell function and metabolism. $\mathbf{b}$ Venn diagram representation of the subsets of Raptor-regulated genes in 2-week-old purified $\beta$-cells that were enriched in $\alpha$-cells and heatmap showed the differential expression of nine overlapped genes in 2-week-old WT and $\beta$ RapKOGFP $\beta$-cells $(n=3-4)$. c Analysis strategy to identify Raptor-dependent genes in $\beta$-cells is shown. $\mathbf{d}$ Heatmap of seven Raptor-dependent genes obtained from 8-week-old RNA-seq and 2-week-old microarray. e Volcano plot shows differential genes between 2-week-old WT and $\beta$ RapKOGFP $\beta$-cells. Microarray identification of Etv1 and Tspan12 as significantly upregulated $\alpha$-cell-enriched genes in 2 -week-old $\beta$ RapKOGFP $\beta$-cells. $\mathbf{f}$ INS-1 cells were transfected with SiRaptor or SiNC for $48 \mathrm{~h}$. qRT-PCR confirmed Raptor-dependent genes in INS-1 cells ( $n=4$ independent cell experiments, for Raptor, $p=0.029$; for Slc2a2, $p=0.048$; for MsIn, $p=0.031$; for Ppp1r1a, $p=0.28$; for Etv1, $p=0.004 ;$ for Tspan12, $p=0.008$; for Aass, $p=0.02$ ). $\mathbf{g}$, $\mathbf{h}$ INS-1 cells were transfected with GFP, Etv1, or Tspan12 overexpression vector. $\mathbf{g}$ Luciferase reporter assay using a rat glucagon promoter reporter ( $n=3$ independent cell experiments, $p=0$.039). h qRT-PCR analysis of glucagon expression in vector transfected INS-1 cells ( $n=4$ independent cell experiments, $p=0.02$ ). Data represent means \pm SEM. ${ }^{\star} p<0.05,{ }^{\star \star} p<0.01$ by two-sided Student's $t$-test.

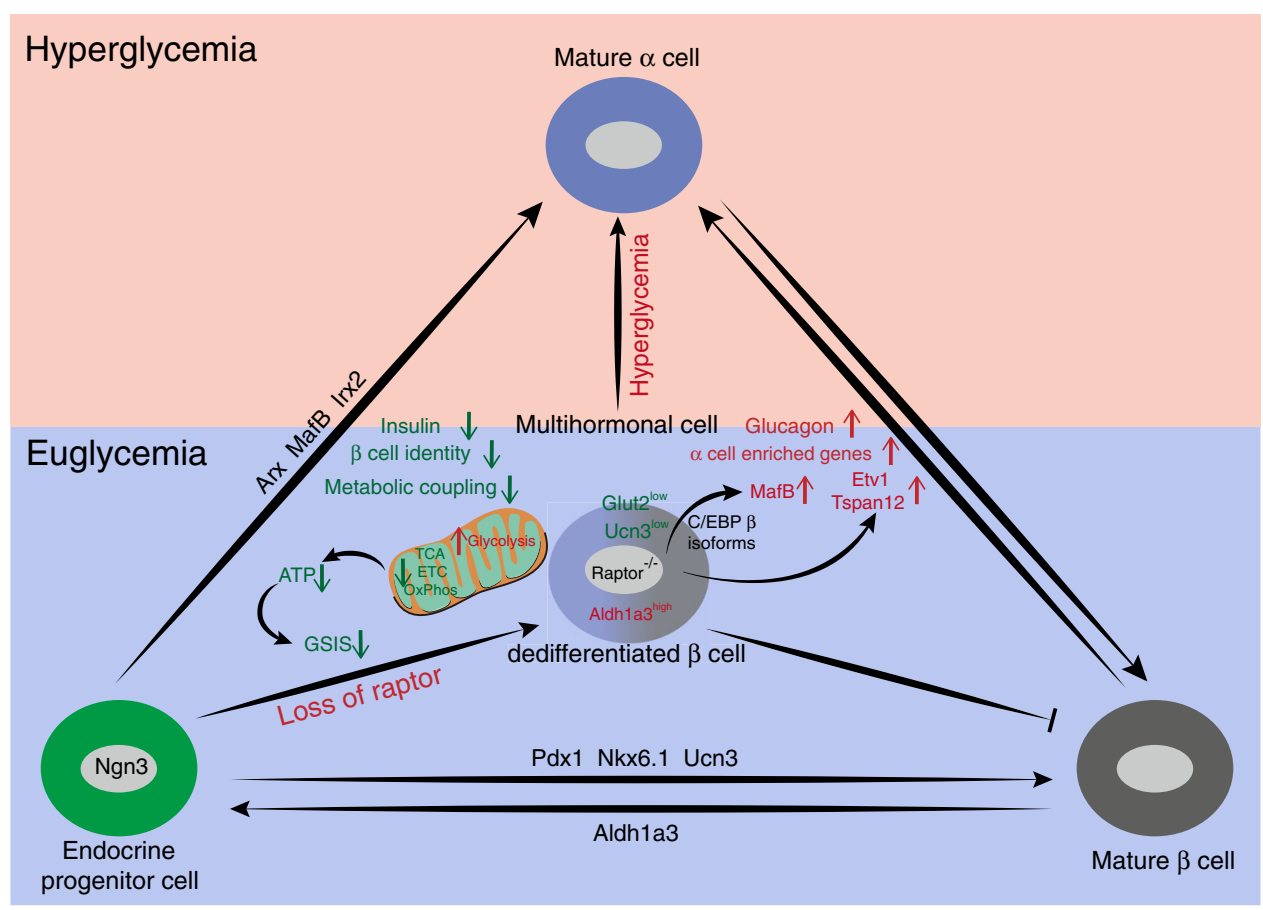

Fig. 7 The role of Raptor in $\boldsymbol{\beta}$-cell identity maintenance and $\boldsymbol{\alpha}$-cell gene repression. Raptor is required for maintaining $\beta$-cell identity as well as repressing $\alpha$-cell signature. At euglycemia, loss of Raptor results in $\beta$-cell dedifferentiation (marked as UCN3low, Glut2low, and Aldh1a3 high) with compromised $\beta$-cell identity, metabolic uncoupling, and regression to multi-hormonal state with $\alpha$-cell features. We propose Raptor/C/EBP $\beta /$ MafB-dependent and MafBindependent ways in silencing $\alpha$-cell-enriched genes and glucagon expression in healthy $\beta$-cells. Raptor-deficient $\beta$-cells with compromised identity become dysfunctional and eventually cause diabetes; in turn, hyperglycemia further aggravates dedifferentiation and reprogramming process.

chemiluminescence (Millipore, USA) and detection was performed using LAS-4000 (GE, USA) and Image Studio Software version 5.2 (LI-COR).

In vitro GSIS and proinsulin/C-peptide measurement. Isolated islets were recovered in 1640 RPMI supplemented with $10 \%$ serum at a $11.1 \mathrm{mM}$ glucose. Then the islets were pre-incubated for $1 \mathrm{~h}$ at $37^{\circ} \mathrm{C}$ in Krebs-Ringer bicarbonate HEPES buffer (KRBH) supplemented with $0.2 \%$ BSA in the presence of $2.8 \mathrm{mM}$ glucose, followed by $1 \mathrm{~h}$ of incubation in KRBH supplemented with $0.2 \%$ BSA in the presence of $2.8 \mathrm{mM}$ glucose or $16.7 \mathrm{mM}$ glucose. The supernatant was collected and C-peptide content was extracted by acid ethanol. C-peptide in supernatant and islet lysates were measured by C-peptide ELISA assay (Mouse C-Peptide ELISA Kit, Crystal Chem, USA). Secreted C-peptide was calculated as the percentage of total C-peptide content per hour. Islet proinsulin and C-peptide content were measured using the islet lysates by Proinsulin (Rat/Mouse Proinsulin ELISA, Mercodia, Sweden) and C-peptide (Mouse C-Peptide ELISA Kit, Crystal Chem, USA) ELISA kits.

ATP levels were measured using the Bioluminescence assay kit (FLASC, Sigma, USA). For ATP measurement, batches of 30 islets each condition following the procedure of GSIS were lysed using the kit buffer at $25^{\circ} \mathrm{C}$ for $5 \mathrm{~min}$. ATP was expressed as relative light units using a luminometer (Promega, Madison, WI).

INS-1 cell culture and luciferase assays. INS-1 cells purchased from the CAMS Cell Culture Center (Beijing, China) were grown in RPMI 1640 medium containing $11.1 \mathrm{mM}$ glucose and supplemented with $10 \mathrm{mM}$ HEPES, $10 \% \mathrm{FBS}, 2 \mathrm{mM} \mathrm{L-}$ glutamine, $1 \mathrm{mM}$ sodium pyruvate, $5 \mu \mathrm{M} \beta$-mercaptoethanol, $100 \mathrm{IU} / \mathrm{mL}$ penicillin and $100 \mu \mathrm{g} / \mathrm{mL}$ streptomycin at $37^{\circ} \mathrm{C}$ in a humidified $5 \% \mathrm{CO}_{2}$ atmosphere. The cells were sub-cultured when they reached $80 \%$ confluence. PcDNA3.1-LAP plasmid, pcDNA3.1-LIP plasmid, pcDNA3.1-Etv1 plasmid, and pcDNA3.1-Tspan12 plasmid transfections were conducted using Lipofectamine 2000 (Invitrogen, USA) according to the manufacturer's instructions.

SiRNA oligonucleotides for Raptor, MafB, and their control were designed and synthesized from GenePharma (Shanghai, China). Lipofectamine 2000 transfection reagent (Invitrogen, USA) was used according to the manufacturer's instructions.

INS-1 cells transfected with LAP/LIP, Etv1/Tspan12 plasmid, or GFP plasmid were plated in 24-well plates $24 \mathrm{~h}$ before transfection. When the cells reached $70-80 \%$ confluence, they were transfected with plasmids (examined promoter plasmid and pRL-SV40) using Lipofectamine 2000 transfection reagent (Invitrogen, USA). Six hours after transfection, the medium was changed. MafB promoter activity was examination after $12 \mathrm{~h}$, and Glucagon promoter activity was determined $24 \mathrm{~h}$ later. The cells were lysed in $1 \times$ PLB, and luciferase assays were performed using the Dual-Luciferase Reporter Assay System (Promega, USA) as recommended by the manufacturer. All luciferase assay experiments were performed in triplicate.

Data analysis. Data are presented as means \pm SEM and analyzed by two-tailed Student's $t$-test for two groups and ANOVA analysis for multiple groups. Statistics were computed by SPSS 22.0 (USA). $P$ value less than 0.05 was considered to be statistically significant. 
Reporting summary. Further information on research design is available in the Nature Research Reporting Summary linked to this article.

\section{Data availability}

All data supporting the findings of this study are available with the article. Data from RNA-seq are available under GEO Series GSE130792. Microarray data has been deposited in the NCBI GEO repository with an accession ID GSE140224. Source data for Figs. 1-6 and Supplementary Figs. 1-4, Supplementary Figs. 6 and 7 are provided with the paper as a Source Data File.

Received: 19 April 2019; Accepted: 5 March 2020;

Published online: 21 May 2020

\section{References}

1. Weir, G. C. \& Bonner-Weir, S. Five of stages of evolving $\beta$-cell dysfunction during progression to diabetes. Diabetes 53, S16-S21 (2004).

2. Accili, D. et al. When $\beta$-cells fail: lessons from dedifferentiation. Diabetes Obes. Metab. 18, 117-122 (2016).

3. Remedi, M. S. \& Emfinger, C. Pancreatic $\beta$-cell identity in diabetes. Diabetes Obes. Metab. 18, 110-116 (2016).

4. Brereton, M. F., Rohm, M. \& Ashcroft, F. M. $\beta$-Cell dysfunction in diabetes: a crisis of identity? Diabetes Obes. Metab. 18, 102-109 (2016).

5. Talchai, C., Xuan, S., Lin, H. V., Sussel, L. \& Accili, D. Pancreatic $\beta$ cell dedifferentiation as a mechanism of diabetic $\beta$ cell failure. Cell 150, 1223-1234 (2012).

6. Gao, T. et al. Pdxl maintains $\beta$ cell identity and function by repressing an $\alpha$ cell program. Cell Metab. 19, 259-271 (2014).

7. Taylor, B., Liu, F. \& Sander, M. Nkx6.1 is essential for maintaining the functional state of pancreatic $\beta$ cells. Cell Rep. 4, 1262-1275 (2013).

8. Nishimura, W., Takahashi, S. \& Yasuda, K. MafA is critical for maintenance of the mature $\beta$ cell phenotype in mice. Diabetologia 58, 566-574 (2014).

9. Gutiérrez, G. D. et al. Pancreatic $\beta$ cell identity requires continual repression of non- $\beta$ cell programs. J. Clin. Invest. 127, 244-259 (2017).

10. Swisa, A. et al. Pax6 maintains pancreatic $\beta$ cell identity by repressing alternative islet cell genes. J. Clin. Invest. 127, 230-243 (2017).

11. Accili, D. Insulin action research and the future of diabetes treatment: the 2017 Banting Medal for Scientific Achievement Lecture. Diabetes 67, 1701-1709 (2018).

12. Brereton, M. F. et al. Reversible changes in pancreatic islet structure and function produced by elevated blood glucose. Nat. Commun. 5, 1-11 (2014).

13. Wang, Z., York, N. W., Nichols, C. G. \& Remedi, M. S. Pancreatic $\beta$ cell dedifferentiation in diabetes and redifferentiation following insulin therapy. Cell Metab. 19, 872-882 (2014).

14. Sun, J. et al. Beta-cell dedifferentiation in patients with T2D with adequate glucose control and nondiabetic chronic pancreatitis. J. Clin. Endocrinol. Metab. 104, 83-94 (2019).

15. Saxton, R. A. \& Sabatini, D. M. mTOR signaling in growth, metabolism, and disease. Cell 168, 960-976 (2017).

16. Kim, D. H. et al. mTOR interacts with raptor to form a nutrient-sensitive complex that signals to the cell growth machinery. Cell 110, 163-175 (2002).

17. Hara, K. et al. Raptor, a binding partner of target of rapamycin (TOR), mediates TOR action. Cell 110, 177-189 (2002).

18. Kim, D. et al. G $\beta \mathrm{L}$, a positive regulator of the rapamycin-sensitive pathway required for the nutrient-sensitive interaction between Raptor and mTOR. Mol. Cell 11, 895-904 (2003).

19. Yuan, T. et al. Reciprocal regulation of mTOR complexes in pancreatic islets from humans with type 2 diabetes. Diabetologia 60, 668-678 (2017).

20. Sacco, F. et al. Phosphoproteomics reveals the GSK3-PDX1 axis as a key pathogenic signaling node in diabetic islets. Cell Metab. 29,1422-1432.e3 (2019).

21. Blandino-Rosano, $M$. et al. mTORC1 signaling and regulation of pancreatic $\beta$ cell mass. Cell Cycle 11, 1892-1902 (2012).

22. Ardestani, A., Lupse, B., Kido, Y., Leibowitz, G. \& Maedler, K. MTORC1 signaling: a double-edged sword in diabetic $\beta$ cells. Cell Metab. 27, 314-331 (2018).

23. Bartolomé, A. et al. Pancreatic $\beta$-cell failure mediated by mTORC1 hyperactivity and autophagic impairment. Diabetes 63, 2996-3008 (2014).

24. Mori, H. et al. Critical roles for the TSC-mTOR pathway in $\beta$-cell function. AJP Endocrinol. Metab. 297, E1013-E1022 (2009).

25. Ni, Q. et al. Raptor regulates functional maturation of murine beta cells. Nat. Commun. 8, 15755 (2017).

26. Blandino-Rosano, $M$. et al. Loss of mTORC1 signalling impairs $\beta$-cell homeostasis and insulin processing. Nat. Commun. 8, 1-15 (2017).
27. Poitout, V. \& Robertson, R. P. Glucolipotoxicity: fuel excess and $\beta$-cell dysfunction. Endocr. Rev. 29, 351-366 (2008)

28. Blum, B. et al. Functional beta-cell maturation is marked by an increased glucose threshold and by expression of urocortin 3. Nat. Biotechnol. 30, 261-264 (2012).

29. van der Meulen, T. et al. Urocortin 3 marks mature human primary and embryonic stem cell-derived pancreatic alpha and beta cells. PLoS ONE 7, 1-12 (2012).

30. Pullen, T. J., Huising, M. O. \& Rutter, G. A. Analysis of purified pancreatic islet beta and alpha cell transcriptomes reveals 11beta-hydroxysteroid dehydrogenase (Hsd11b1) as a novel disallowed gene. Front. Genet. 8, 41 (2017).

31. Qiu, W. L. et al. Deciphering pancreatic islet $\beta$ cell and a cell maturation pathways and characteristic features at the single-cell level. Cell Metab. 27, 702 (2018).

32. Cigliola, V. et al. Pancreatic islet-autonomous insulin and smoothenedmediated signalling modulate identity changes of glucagon $+\alpha$-cells. Nat. Cell Biol. 20, 1267-1277 (2018).

33. Artner, I. et al. An activator of the glucagon gene expressed in developing islet $\alpha$ - and $\beta$-cells. Diabetes 55, 297-304 (2006).

34. Jeong, H.-W. et al. Transcriptional regulation of endothelial cell behavior during sprouting angiogenesis. Nat. Commun. 8, 726 (2017).

35. Soucie, E. L. et al. Lineage-specific enhancers activate self-renewal genes in macrophages and embryonic stem cells. Science 351, aad5510 (2016).

36. Rosen, E. D. \& MacDougald, O. A. Adipocyte differentiation from the inside out. Nat. Rev. Mol. Cell Biol. 7, 885-896 (2006).

37. Descombes, P. \& Schibler, U. A liver-enriched transcriptional activator protein, LAP, and a transcriptional inhibitory protein, LIP, are translated from the same mRNA. Cell 67, 569-579 (1991).

38. White, M. G. et al. Expression of mesenchymal and $\alpha$-cell phenotypic markers in Islet $\beta$-cells in recently diagnosed diabetes. Diabetes Care 36, 3818-3820 (2013).

39. Yoneda, $\mathrm{S}$. et al. Predominance of $\beta$-cell neogenesis rather than replication in humans with an impaired glucose tolerance and newly diagnosed diabetes. $J$. Clin. Endocrinol. Metab. 98, 2053-2061 (2013).

40. Calkhoven, C. F., Mu, C. \& Leutz, A. Translational control of C/EBP alpha and C/EBP beta isoform expression. Genes Dev. 14, 1920-1932 (2000).

41. Smink, J. J. et al. Transcription factor $\mathrm{C} / \mathrm{EBP} \beta$ isoform ratio regulates osteoclastogenesis through MafB. EMBO J. 28, 1769-1781 (2009).

42. Lu, B. C. et al. Etv4 and Etv5 are required downstream of GDNF and Ret for kidney branching morphogenesis. Nat. Genet. 41, 1295-1302 (2009).

43. Mao, J., McGlinn, E., Huang, P., Tabin, C. J. \& McMahon, A. P. Fgfdependent Etv4/5 activity is required for posterior restriction of Sonic Hedgehog and promoting outgrowth of the vertebrate limb. Dev. Cell 16, 600-606 (2009)

44. Zhang, Z., Verheyden, J. M., Hassell, J. A. \& Sun, X. FGF-regulated Etv genes are essential for repressing Shh expression in mouse limb buds. Dev. Cell 16 607-613 (2009).

45. Junge, H. J. et al. TSPAN12 regulates retinal vascular development by promoting Norrin- but not Wnt-induced FZD4/beta-catenin signaling. Cell 139, 299-311 (2009).

46. Charrin, S., Jouannet, S., Boucheix, C. \& Rubinstein, E. Tetraspanins at a glance. J. Cell Sci. 127, 3641-3648 (2014).

47. Hemler, M. E. Tetraspanin proteins promote multiple cancer stages. Nat. Rev Cancer 14, 49-60 (2014).

48. Suriben, R. et al. Beta-cell insulin secretion requires the ubiquitin ligase COP1. Cell 163, 1457-1467 (2015).

49. Dhawan, S. et al. Pancreatic $\beta$ cell identity is maintained by DNA methylationmediated repression of Arx. Dev. Cell 20, 419-429 (2011).

50. Ferrante, A. W. et al. Metabolic inflexibility impairs insulin secretion and results in MODY-like diabetes in triple FoxO-deficient mice. Cell Metab. 20, 593-602 (2014).

51. Becker, T. C., BeltrandelRio, H., Noel, R. J., Johnson, J. H. \& Newgard, C. B. Overexpression of hexokinase I in isolated islets of Langerhans via recombinant adenovirus. Enhancement of glucose metabolism and insulin secretion at basal but not stimulatory glucose levels. J. Biol. Chem. 269, 21234-21238 (1994)

52. Gerst, F. et al. The expression of aldolase B in islets is negatively associated with insulin secretion in humans. J. Clin. Endocrinol. Metab. 103, 4373-4383 (2018).

53. Sekine, N. et al. Low lactate dehydrogenase and high mitochondrial glycerol phosphate dehydrogenase in pancreatic beta-cells. Potential role in nutrient sensing. J. Biol. Chem. 269, 4895-4902 (1994).

54. Sikström, K. et al. mTORC1 controls mitochondrial activity and biogenesis through 4E-BP-dependent translational regulation. Cell Metab. 18, 698-711 (2013).

55. Siddiqui, N. et al. mTOR controls mitochondrial dynamics and cell survival via MTFP1. Mol. Cell 67, 922-935.e5 (2017). 
56. Kim-Muller, J. Y. et al. Aldehyde dehydrogenase 1a3 defines a subset of failing pancreatic $\beta$ cells in diabetic mice. Nat. Commun. 7, 1-11 (2016).

57. Alarcon, C. et al. Pancreatic $\beta$-cell adaptive plasticity in obesity increases insulin production but adversely affects secretory function. Diabetes $\mathbf{6 5}$, 438-450 (2016).

58. Rhodes, C. J. \& Alarcon, C. What beta-cell defect could lead to hyperproinsulinemia in NIDDM? Some clues from recent advances made in understanding the proinsulin-processing mechanism. Diabetes 43, 511-517 (1994).

59. Navarro-González, J. F., Mora-Fernández, C., De Fuentes, M. M. \& GarcíaPérez, J. Inflammatory molecules and pathways in the pathogenesis of diabetic nephropathy. Nat. Rev. Nephrol. 7, 327-340 (2011).

60. Sun, Q., Li, J. \& Gao, F. New insights into insulin: the anti-inflammatory effect and its clinical relevance. World J. Diabetes 5, 89 (2017).

61. Anupam, K., Kaushal, J., Prabhakar, N. \& Bhatnagar, A. Effect of redox status of peripheral blood on immune signature of circulating regulatory and cytotoxic $\mathrm{T}$ cells in streptozotocin induced rodent model of type I diabetes. Immunobiology 223, 586-597 (2018).

62. Bayrami, G. et al. Combination of Vildagliptin and ischemic postconditioning in diabetic hearts as a working strategy to reduce myocardial reperfusion injury by restoring mitochondrial function and autophagic activity. Adv. Pharm. Bull. 8, 319-329 (2018).

63. Guo, $S$. et al. Inactivation of specific $\beta$ cell transcription factors in type 2 diabetes. J. Clin. Invest. 123, 3305-3316 (2013).

64. Zraika, S. et al. Oxidative stress is induced by islet amyloid formation and time-dependently mediates amyloid-induced beta cell apoptosis. Diabetologia 52, 626-635 (2009).

65. Gu, Y., Lindner, J., Kumar, A., Yuan, W. \& Magnuson, M. A. Rictor/mTORC2 is essential for maintaining a balance between $\beta$-cell proliferation and cell size. Diabetes 60, 827-837 (2011).

66. Kitamura, K. et al. Mutation of ARX causes abnormal development of forebrain and testes in mice and X-linked lissencephaly with abnormal genitalia in humans. Nat. Genet. 32, 359-369 (2002).

67. Trapnell, C., Pachter, L. \& Salzberg, S. L.TopHat: discovering splice junctions with RNA-Seq. Bioinformatics 25 (9):1105-1111

68. Trapnell, C. et al. Transcript assembly and abundance estimation from RNASeq reveals thousands of new transcripts and switching among isoforms. Nat. Biotechnol. 28, 511-515 (2011).

69. Zhu, R. et al. Defective endoplasmic reticulum export causes proinsulin misfolding in pancreatic beta cells. Mol. Cell. Endocrinol. 493, 110470 (2019).

\section{Acknowledgements}

This work was supported by the National Natural Sciences Foundation of China Grants $(81870527,81670700)$ as well as the Shanghai Municipal Education Commission-

Gaofeng Clinical Medicine Grant Support 20172006 (to Q.W.). We thank Prof D. Accili (Naomi Berrie Diabetes Center and Departments of Medicine, Columbia University) for the helpful discussion and valuable advice. We thank Juan Chen (State Key Laboratory for Medical Genomics, Shanghai Institute of Hematology and Collaborative Innovation Center of Hematology, Rui-Jin Hospital affiliated to Shanghai Jiao Tong University
School of Medicine, Shanghai) for the technical assistance for FACS analysis and sorting GFP labeled $\beta$-cells. We also thank Qin Hang (Core Facility of Basic Medical Science of SJTU) and Jie Yang (Core Facility of Basic Medical Science of SJTU) for their technical supports with confocal microscopy and TEM imaging. We are grateful to Dr. Kunio Kitamura (Mitsubishi Kagaku Institute of Life Sciences) for kindly providing anti-Arx antibody and Prof. Ming Liu (Department of Endocrinology and Metabolism, Tianjin Medical University General Hospital, Tianjin, China) for the gift of anti-proinsulin antibody.

\section{Author contributions}

Q.Y., Q.N., Y.W., H.Z., W.L., and A.N. performed experiments and analyzed the data. Q. Y., Q.N., Y.W., and Q.W. wrote the manuscript. S.W. and Y.G. provided helpful discussions. Q.W. and G.N. designed the project, supervised the research, and coordinated the execution of the experimental plan.

\section{Competing interests}

The authors declare no competing interests.

\section{Additional information}

Supplementary information is available for this paper at https://doi.org/10.1038/s41467020-15935-0.

Correspondence and requests for materials should be addressed to Q.W. or G.N.

Peer review information Nature Communications thanks Guy Rutter and the other, anonymous, reviewer(s) for their contribution to the peer review of this work.

Reprints and permission information is available at http://www.nature.com/reprints

Publisher's note Springer Nature remains neutral with regard to jurisdictional claims in published maps and institutional affiliations.

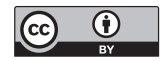

Open Access This article is licensed under a Creative Commons Attribution 4.0 International License, which permits use, sharing, adaptation, distribution and reproduction in any medium or format, as long as you give appropriate credit to the original author(s) and the source, provide a link to the Creative Commons license, and indicate if changes were made. The images or other third party material in this article are included in the article's Creative Commons license, unless indicated otherwise in a credit line to the material. If material is not included in the article's Creative Commons license and your intended use is not permitted by statutory regulation or exceeds the permitted use, you will need to obtain permission directly from the copyright holder. To view a copy of this license, visit http://creativecommons.org/ licenses/by/4.0/

(C) The Author(s) 2020 